\title{
Experimental Glucocorticoid Receptor Agonists for the Treatment of Asthma: A Systematic Review
}

This article was published in the following Dove Press journal:

Journal of Experimental Pharmacology

\author{
Paola Rogliani (D) ${ }^{1,2}$ \\ Beatrice Ludovica Ritondo' \\ Ermanno Puxeddu ${ }^{2}$ \\ Gloria Pane 2 \\ Mario Cazzola (iD ${ }^{\prime}$ \\ Luigino Calzetta iD $^{3}$ \\ 'Unit of Respiratory Medicine, \\ Department of Experimental Medicine, \\ University of Rome "Tor Vergata", Rome, \\ Italy; 'Division of Respiratory Medicine, \\ University Hospital "Tor Vergata", Rome, \\ Italy; ${ }^{3}$ Department of Medicine and \\ Surgery, Respiratory Disease and Lung \\ Function Unit, University of Parma, \\ Parma, Italy
}

\begin{abstract}
Inhaled corticosteroids (ICSs) are considered the cornerstone of asthma treatment. Despite the solid evidence documenting the efficacy and safety of ICSs at the level of the airways, their use can be affected by pulmonary and systemic adverse events (AEs) when administered chronically and/or at high doses. Thus, there is a pharmacological and medical need for new glucocorticoid (GC) receptor (GR) ligands with a more favorable therapeutic index, in order to overcome the shortcomings of currently available ICSs. The therapeutic profile of GCs can be improved by enhancing genomic mechanisms mediated by transrepression, which is assumed to be responsible for several anti-inflammatory and immunomodulatory actions, rather than transactivation, which causes most of the GC-associated AEs. It was assumed that an independent modulation of the molecular mechanisms underlying transactivation and transrepression could translate into the dissociation of beneficial effects from AEs. Therefore, current research is looking for GCs that are able to elicit prevalently transrepression with negligible transactivating activity. These compounds are known as selective glucocorticoid receptor agonists (SEGRAs). In this review, experimental GR agonists currently in pre-clinical and clinical development for the treatment of asthma have been systematically assessed. Several compounds are currently under pre-clinical development, but only three novel experimental GR agonists (GW870086X, AZD5423, AZD7594) seem to have some potential therapeutic relevance and have entered clinical trials for the treatment of asthma. Since data from pre-clinical studies have not always been confirmed in clinical investigations, well-designed randomized controlled trials are needed in asthmatic patients to confirm the potentially positive benefit/risk ratio of each specific SEGRA and to optimize the development strategy of these agents in respiratory medicine.

Keywords: asthma, glucocorticoid agonists, SEGRA, efficacy, safety
\end{abstract}

\section{Introduction}

Asthma is a chronic inflammatory disorder of the airways affecting more than 300 million people worldwide, and is associated with a huge social and economic burden, estimated to rank as one of the highest among chronic illnesses. ${ }^{1}$ According to the World Health Organization, asthma causes 250,000 deaths annually worldwide, and it is responsible for more than 500,000 hospitalizations every year in the USA. $^{2}$

Inhaled corticosteroids (ICSs) are considered the cornerstone of asthma treatment, and are administered alone or in combination with long-acting $\beta_{2}$-adrenoceptor agonists (LABAs) as first-line therapy for persistent asthma. ${ }^{3}$ Despite the solid
Correspondence: Paola Rogliani Department of Experimental Medicine, Via Montpellier I, Rome 00133 , Italy Tel +390620904656

Email paola.rogliani@uniroma2.it

Journal of Experimental Pharmacology 2020:12 233-254 
evidence documenting the efficacy and safety of ICSs at the level of the airways, their use can be affected by pulmonary and systemic adverse events (AEs) when administered chronically and/or at high doses, along with potential steroid resistance. ${ }^{4}$ Therefore, researchers are currently looking for new glucocorticoid (GC) receptor (GR) ligands with a more favorable therapeutic index, in order to overcome the shortcomings of currently available ICSs. ${ }^{5}$

The therapeutic profile of GCs can be improved by enhancing genomic mechanisms mediated by transrepression, which is assumed to be responsible for several antiinflammatory and immunomodulatory actions by inhibiting the expression of cytokines and other pro-inflammatory molecules, rather than transactivation, which causes most of the GC-associated AEs. ${ }^{6}$ However, transactivation may also modulate some therapeutic effects, leading to the expression of anti-inflammatory and regulator proteins. ${ }^{7}$ It

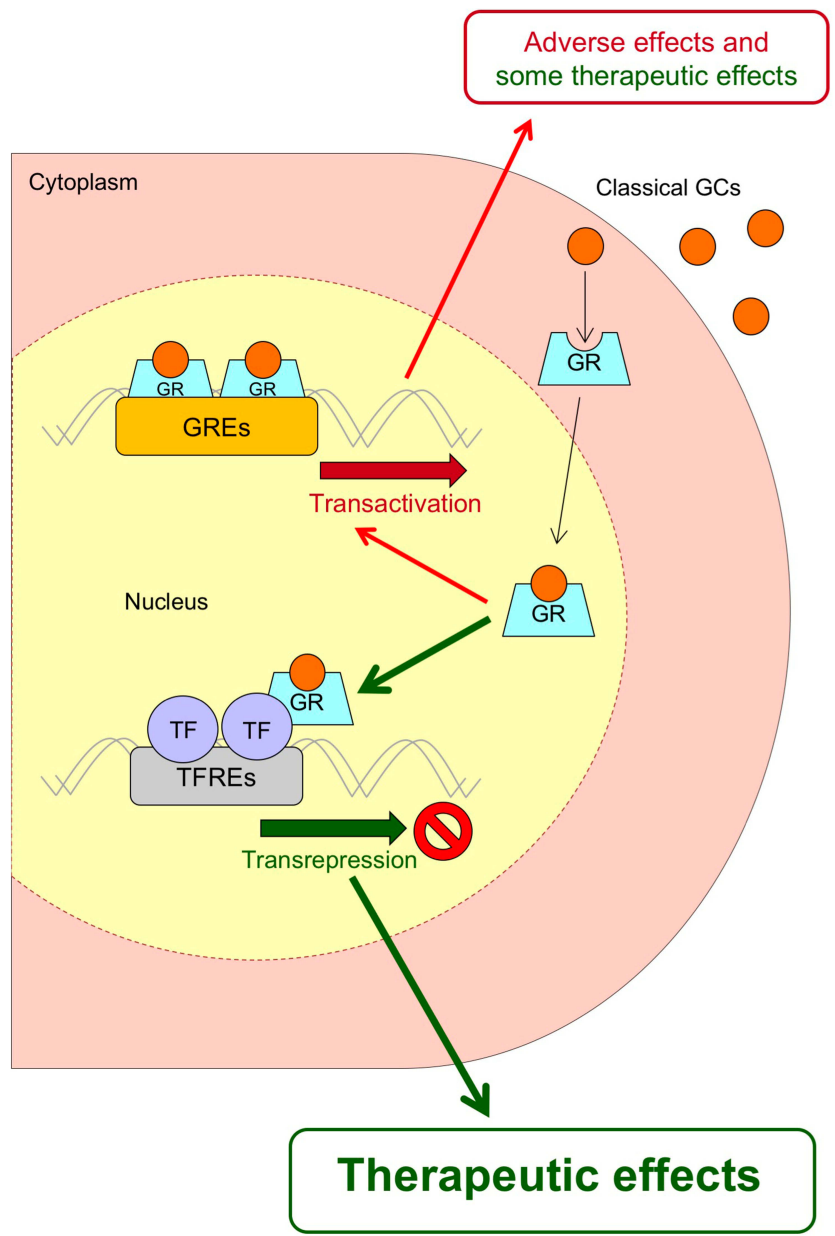

was assumed that an independent modulation of the molecular mechanisms underlying transactivation and transrepression could translate into the dissociation of beneficial effects from AEs. Therefore, the main goal of research is to identify GCs that are able to elicit prevalently transrepression with negligible transactivating activity. ${ }^{8}$ These compounds are known as selective glucocorticoid receptor agonists (SEGRAs), selective glucocorticoid receptor modulators (SEGRMs), or dissociated GR ligands, and they are able to activate specific GR mechanisms and alter GRmediated gene expression (Figure 1). ${ }^{9}$

At the beginning, these ligands were called SEGRAs, since they were developed from a steroidal scaffold and often displayed a partial agonistic activity on transactivation. ${ }^{9}$ The term SEGRM is more recent, and used in order to differentiate the more recent nonsteroidal compounds from the older agents with an

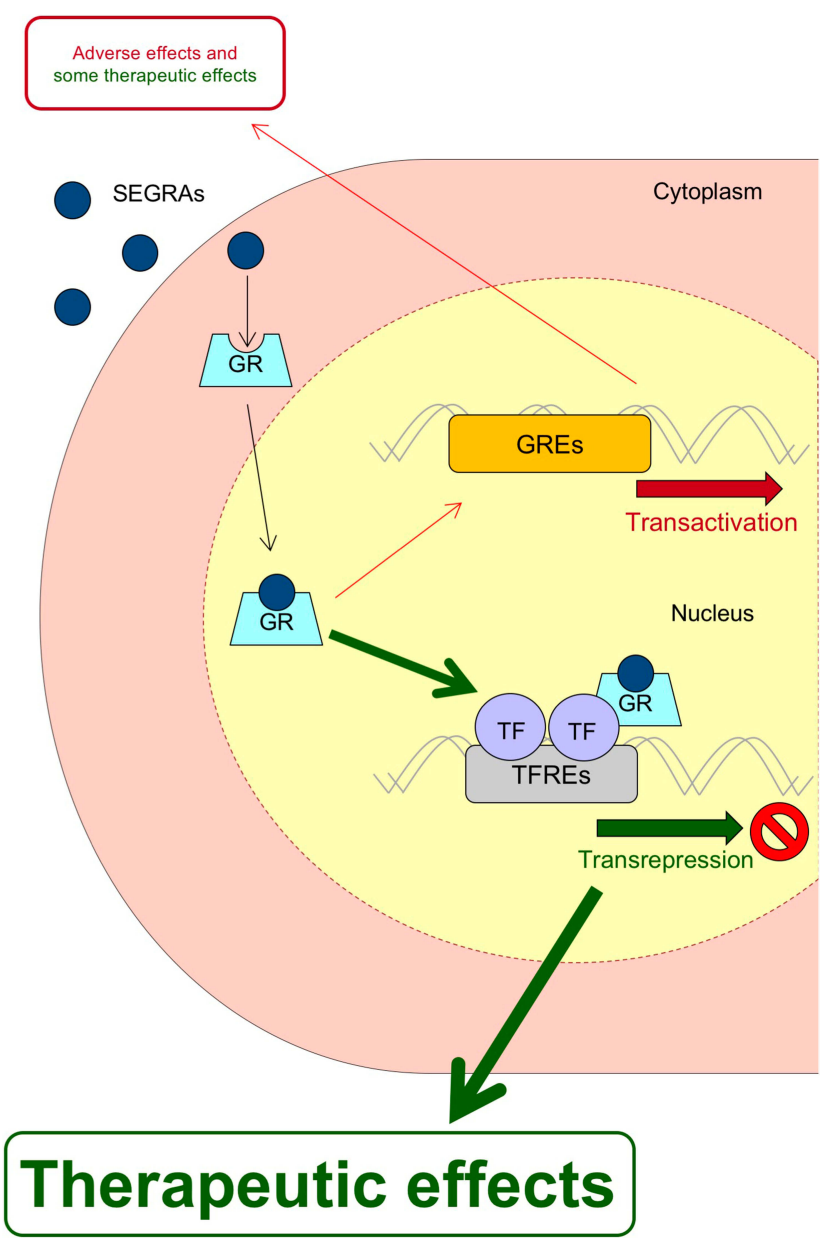

Figure I Model of GR transactivation and transrepression of genes, when induced by classical GCs and SEGRAs. SEGRAs have been designed to improve the therapeutic index compared to classical GCs, by preferentially inducing transrepression with negligible transactivating activity.

Abbreviations: GC, glucocorticoid; GR, glucocorticoid receptor; GRE, glucocorticoid response element; SEGRA, selective glucocorticoid receptor agonist; TF, transcription factor; TFRE, transcription factor responsive element. 
improved therapeutic ratio, and/or to identify GR ligands able to modulate the activity of a GR agonist and that may not classically bind to the receptor pocket. ${ }^{9,10}$

The aim of this review was to systematically assess the experimental GR agonists currently in pre-clinical and clinical development for the treatment of asthma.

\section{Materials and Methods Review Question}

The question investigated by this systematic review was whether the current experimental GR agonists might be suitable for an effective and safe treatment of patients with asthma.

\section{Search Strategy}

The protocol of this qualitative synthesis of the current literature has been submitted to the international prospective register of systematic reviews (PROSPERO, submission ID: 171,465), and performed in agreement with the Preferred Reporting Items for Systematic Reviews and Meta-Analyses Protocols (PRISMA-P), ${ }^{11}$ with the relative flow diagram reported in Figure 2. This study satisfied all the recommended items reported by the PRISMA-P checklist. ${ }^{11}$

The PICO (Patient problem, Intervention, Comparison, and Outcome) framework was applied to develop the literature search strategy and question, as previously reported. ${ }^{12}$ Namely, the "Patient problem" included patients suffering from asthma; the "Intervention" regarded experimental GR agonists; the "Comparison" was performed with active comparators and/or placebo; and the assessed "Outcomes" were the activity in vitro, lung function, use of rescue medications, asthma symptom control, pharmacokinetic (PK) and pharmacodynamic (PD) characteristics, and safety profile.

In this study, experimental GR agonists are considered investigational medications, which in turn are defined as drugs that have received approval by either the US Food and Drug Administration or the European Medicines Agency for human testing but that are not approved for marketing. ${ }^{13,14}$

A comprehensive literature search was performed for in vitro studies, in vivo studies performed on laboratory animals, and clinical trials, written in English and investigating the impact of experimental GR agonists for the

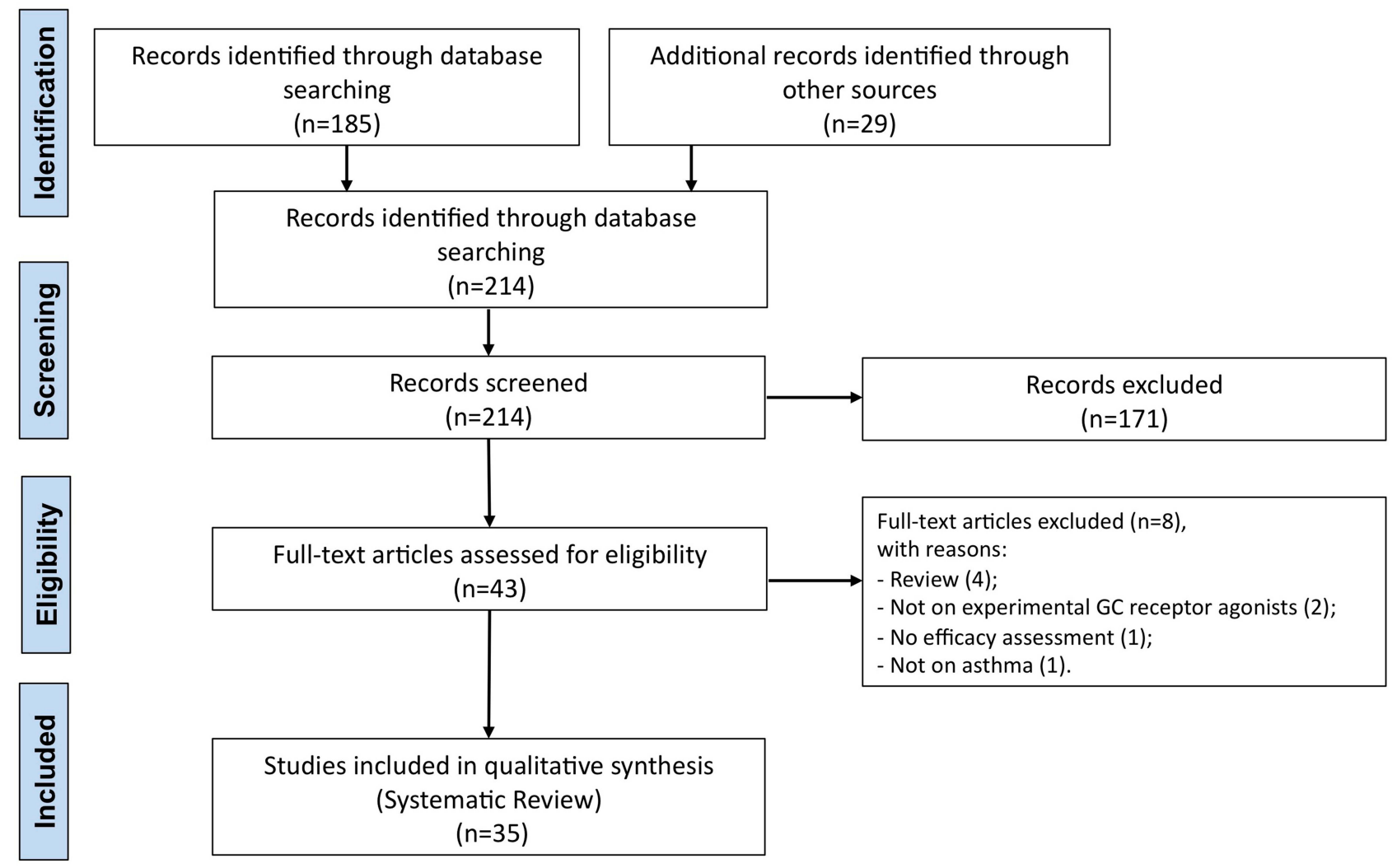

Figure 2 PRISMA flow diagram for the identification of the studies included in the systematic review concerning the impact of experimental GR agonists for the treatment of asthma.

Abbreviations: GC, glucocorticoid; GR, glucocorticoid receptor; PRISMA, Preferred Reporting Items for Systematic Reviews and Meta-Analyses. 
treatment of asthma. The search was performed in ClinicalTrials.gov, Cochrane Central Register of Controlled Trials, Embase, EU Clinical Trials Register, MEDLINE, Scopus, and Web of Science in order to provide for relevant studies available with no time limit up to April 30th, 2020. The searched terms were "glucocorticoid receptor agonists AND asthma", and the research string was as follows: (("receptors, glucocorticoid" [MeSH Terms] OR ("receptors"[All Fields] AND "glucocorticoid"[All Fields]) OR "glucocorticoid receptors"[All Fields] OR ("glucocorticoid"[All Fields] AND "receptor"[All Fields]) OR ("glucocorticoid receptor"[All Fields]) AND ("agonists"[Subheading] OR "agonists"[All Fields]) AND ("asthma"[MeSH Terms] OR "asthma"[All Fields]). Studies reporting the in vitro profile and the impact of investigational GR agonists on lung function, the use of rescue medications, asthma symptom control, PK and PD characteristics, and safety were included in this systematic review.

Citations in previous published reviews were checked to select further pertinent studies, if any. ${ }^{5,15-18}$

Two reviewers independently checked the relevant studies identified from literature searches obtained from the abovementioned databases. The studies were selected in agreement with previously mentioned criteria, and any difference in opinion about eligibility was resolved by consensus.

\section{Data Extraction}

Data from the included studies were extracted and checked for study references, NCT number identifier, study characteristics, treatments and comparators with doses and regimen of administration, patient characteristics and number of analyzed patients, age, gender, body mass index, serious adverse events (SAEs), forced expiratory volume in 1 second $\left(\mathrm{FEV}_{1}\right), \mathrm{PK}$ and $\mathrm{PD}$ characteristics, use of rescue medication, asthma symptom control, and Jadad score.

\section{Endpoints}

The co-primary endpoints of this systematic review were the in vitro activity of experimental GR agonists and their impact on $\mathrm{FEV}_{1}$, peak expiratory flow (PEF), fraction exhaled of nitric oxide $\left(\mathrm{F}_{\mathrm{E}} \mathrm{NO}\right)$, use of rescue medication, PK characteristics, and safety profile.

\section{Strategy for Data Synthesis}

Data from original papers were extracted and reported via qualitative synthesis.

\section{Quality of Studies and Risk Bias}

The summary of the risk of bias for each selected randomized controlled trial (RCT) was analyzed via the Cochrane Risk of Bias 2 (RoB 2) and Jadad score. ${ }^{19,20}$

The weighted assessment of the overall risk of bias was analyzed via the Cochrane RoB $2 .^{20}$

The Jadad score, with a scale of 1-5 (score of 5 being the best quality), was used to assess the quality of the clinical trials concerning the likelihood of bias related to randomization, double blinding, withdrawals, and dropouts. ${ }^{19}$ The quality of studies was assessed as follows: total score $\leq 2$, low quality; total score $=3$, medium quality; total score $\geq 4$, high quality.

Two reviewers independently assessed the quality of individual studies, and any difference in opinion about the quality score was resolved by consensus.

\section{Results}

Obtained data were extracted from 35 studies, among which 12 were in vitro studies, ${ }^{21-33}$ five were performed in vivo, ${ }^{34-39}$ three were both in vitro and in vivo, ${ }^{40-42}$ and 15 were $\mathrm{RCTs},{ }^{43-64}$ the characteristics of which are reported in Table 1.

Most RCTs included in this systematic review had a low risk of bias for the missing outcome data and selection of the reported results (100.0\%) and deviation from intended interventions $(53.3 \%)$. The risk of bias for the randomization process was low in $40 \%$ of the RCTs. Some concerns on the risk of bias were detected for the measurement of the outcome $(80.0 \%)$, deviation from intended interventions (46.7\%), and randomization process (40.0\%). A high risk of bias due to randomization process was detected in $13.3 \%$ of the RCTs. The weighted plot for the assessment of the overall risk of bias and the traffic light plot for the assessment of each RCT are reported in Figures 3 and 4, respectively.

Most of the RCTs (60.0\%) included in this systematic review were ranked as being of medium to high quality in agreement with Jadad score, whereas $40.0 \%$ of them were characterized by low quality level (Table 1).

\section{Experimental GR Agonists}

Detailed information on the PK of experimental GR agonists is reported in Table 2.

\section{A222977}

The SEGRA A222977 (also called compound 13), a 5-aryl2,5-dihydro-10-methoxy-2,2,4-trimethyl-1H-[1] 
benzopyrano-[3,4-f]quinolone, is derived from a modified structure of A276575. ${ }^{42,65}$ A222977 exhibited high affinity for GR and low affinity and efficacy for progesterone receptors (PRs), and in a cross-linked Sephadex ${ }^{\circledR}$ dextran gel rat model of asthma with induced eosinophil influx in lung, A222977 per os (PO) was as effective as prednisolone against inflammation. ${ }^{42}$ Despite the promising in vitro profile, no further documented research was performed, perhaps owing to the inability of A222977 and similar molecules to separate the transcriptional repression from the glucocorticoid response element (GRE) transactivator activity. ${ }^{15}$

\section{A276575}

The SEGRA A276575 (2,5-dihydro-9-hydroxy-10methoxy-2,2,4-trimethyl-5-(1-methylcyclohexen-3-y1)1H-[1]benzopyrano[3,4-f]quinoline) has a high transrepression activity and a low GRE transactivation activity and, unlike traditional GCs, it does not contain the terpenoid structure. ${ }^{22}$ A276575 is a 7:1 mixture of syn- and anti-diastereomers. The (-)-enantiomers showed 10-30fold greater affinity for GRs than the respective (+)enantiomers. $^{22}$ Both the (-)-syn and (--)-anti enantiomers were potent inhibitors of prostaglandin $\mathrm{E}_{2}$ release in A549 lung epithelial cells challenged by interleukin-1 $\beta$ (IL-1 $\beta$ ); on the other hand, only the (-)-anti-enantiomer inhibited the production of the chemokine $\mathrm{C}-\mathrm{C}$ motif ligand 5 (CCL5)/regulated on T-cell activation, normal T-cell expressed and secreted (RANTES). ${ }^{22}$

Further clinical development in vivo was precluded, perhaps owing to some properties of A276575 such as a high affinity for PRs and a super-agonistic activity in mouse mammary tumor virus (MMTV) PR-B (MMTV-PR -B) transfection assays, which are undesirable effects not induced by dexamethasone (DEX). ${ }^{22}$

\section{AZD5423}

The SEGRA AZD5423 (2,2,2-trifluoro-N-[(1R,2S)1-[1-(4-fluorophenyl)indazol-5-yl]oxy-1-(3-methoxyphenyl)propan-2-yl]acetamide) is under development for the treatment of asthma. ${ }^{66}$

In transfected pulmonary A549 cells, AZD5423 activated the $2 X G R E$ reporter by inducing a super-agonistic effect on the maximal response $\left(E_{\max }\right)$ relative to DEX and budesonide (BUD). ${ }^{30}$ The effect of AZD5423 was not affected by IL-1 $\beta$, whereas both DEX and BUD reduced $E_{\max }$ in the presence of IL-1 $\beta .^{30}$ AZD5423 was as effective as DEX and BUD in repressing the release of IL-1 $\beta$ induced granulocyte-macrophage colony-stimulating factor (GM-CSF), IL-6, and IL-8, and in inducing TSC22 domain family member 3 (TSC22D3, also known as glucocorticoid-induced leucine zipper), dual-specificity phosphatase-1 (DUSP1, also known as mitogen-activated protein kinase phosphatase 1 [MKP1]), and regulator of G-protein signaling 2 (RGS2) gene expression. ${ }^{30}$

In a Phase I RCT (NCT01225549), nebulized AZD5423 75-300 $\mu \mathrm{g}$, but not BUD, reduced the fall in $\mathrm{FEV}_{1}$ during the late asthmatic response (LAR) compared to placebo (8.7-14.0\%) in mild allergic asthma. No effect was detected on early asthmatic response (EAR). ${ }^{54,55}$ AZD5423 reduced the allergen-induced sputum eosinophilia and airway hyperresponsiveness (AHR) to methacholine compared to placebo. ${ }^{54,55}$

A further three Phase I RCTs evaluated the tolerability of inhaled AZD5423 in healthy volunteers: a singleascending dose study performed mainly on Caucasians, a multiple-ascending dose study, and a single- and multiple-ascending dose trial conducted in Japanese volunteers. ${ }^{50}$ The Caucasian-dominant single-ascending dose RCT documented that AZD5423 8.4-2400 $\mu \mathrm{g}$ via the Spira Electra $2^{\circledR}$ jet nebulizer caused no clinically significant abnormalities in laboratory variables. ${ }^{50}$ AZD5423 was rapidly absorbed in the lung, with a fast initial concentration decrease and slow plasma elimination in the terminal phase. ${ }^{50} \mathrm{PK}$ data from single-ascending dose studies indicated dose-proportional plasma exposure of AZD5423, with no difference between the Caucasiandominant and Japanese data sets. ${ }^{50}$ The repeated-dose plasma exposure investigated in the multiple-ascending dose study confirmed the dose-proportional PK, ${ }^{50}$ with the steady state reached after 7 days. ${ }^{50}$ AZD5423 50-300 $\mu \mathrm{g}$ via a hand-held vibrating mesh nebulizer did not alter laboratory variables, electrocardiogram, vital signs, or spirometry measurements. ${ }^{50}$ According to the two singleascending dose Caucasian-dominant and Japanese studies, accumulation ratio of AZD5423 was low, with no evidence of time-dependent PK. ${ }^{50}$ AZD5423 100-300 $\mu \mathrm{g}$ produced a maximum drug concentration $\left(C_{\max }\right)$ of 4.96-11.1 nmol/L and a time to reach $C_{\max }\left(t_{\max }\right)$ of $0.08-0.1 \mathrm{~h}^{50}$ In the single-ascending dose study performed mainly on Caucasians, AZD5423 500-1248 $\mu \mathrm{g}$ suppressed plasma cortisol compared to placebo. ${ }^{50}$ The maximum reduction in plasma cortisol was detected after a single dose of AZD5423 $1248 \mu \mathrm{g} .{ }^{50}$ In the single- and multiple-ascending dose study conducted in Japanese volunteers, AZD5423 $300 \mu \mathrm{g}$ reduced urinary excretion cortisol. In the multiple-ascending dose study, 
Table I Characteristics of the Clinical Studies Included in the Systematic Review

\begin{tabular}{|c|c|c|c|c|c|c|c|}
\hline $\begin{array}{l}\text { Study and } \\
\text { Year }\end{array}$ & $\begin{array}{l}\text { ClinicalTrials. } \\
\text { gov Identifier } \\
\text { and/or } \\
\text { Company ID }\end{array}$ & $\begin{array}{l}\text { Study } \\
\text { Characteristics }\end{array}$ & $\begin{array}{l}\text { Treatment } \\
\text { Duration } \\
\text { (Days) }\end{array}$ & $\begin{array}{l}\text { Number } \\
\text { of } \\
\text { Analyzed } \\
\text { Patients }\end{array}$ & $\begin{array}{l}\text { Drugs, Doses, } \\
\text { and Regimen of } \\
\text { Administration }\end{array}$ & Comparator & $\begin{array}{l}\text { Route of } \\
\text { Administration }\end{array}$ \\
\hline $\begin{array}{l}\text { Brown et al, } \\
2019^{43,44}\end{array}$ & NCT024794I2 & $\begin{array}{l}\text { Phase lla, multicenter, } \\
\text { randomized, double- } \\
\text { blind, 3-period, } \\
\text { placebo-controlled, } \\
\text { incomplete-block, } \\
\text { crossover }\end{array}$ & 15 & 54 & $\begin{array}{l}\text { AZD7594 } 58 \mu g \text { (q. } \\
\text { d.), } 250 \mu g \text { (q.d.), } \\
800 \mu g \text { (q.d.) }\end{array}$ & Placebo (q.d.) & Oral inhalation \\
\hline $\begin{array}{l}\text { Prothon } \\
\text { et al, } \\
2019^{45,46}\end{array}$ & NCT02645253 & $\begin{array}{l}\text { Phase I, single-center, } \\
\text { randomized, single- } \\
\text { blind, placebo- } \\
\text { controlled, sequential- } \\
\text { group }\end{array}$ & 20 & 27 & $\begin{array}{l}\text { AZD7594 } 200 \mu g \\
\text { (q.d.), } 400 \mu \mathrm{g} \text { (q.d.), } \\
\text { I600 } \mu \text { g (q.d.) }\end{array}$ & Placebo (q.d.) & Oral inhalation \\
\hline $\begin{array}{l}\text { Melin et al, } \\
2017^{47,48}\end{array}$ & $\begin{array}{l}\text { NCT0I310322, } \\
\text { Study A }\end{array}$ & $\begin{array}{l}\text { Phase I, single-center, } \\
\text { partly randomized, } \\
\text { open-label, 4-way, } \\
\text { crossover }\end{array}$ & I & 13 & $\begin{array}{l}\text { AZD5423 } 250 \mu \mathrm{g}, \\
420 \mu \mathrm{g}, 450 \mu \mathrm{g}, \\
\text { I200 } \mu \mathrm{g} \text { (single } \\
\text { dose) }\end{array}$ & NA & $\begin{array}{l}\text { AZD5423 } \\
420 \mu \mathrm{g}, 450 \mu \mathrm{g}: \\
\text { oral inhalation, } \\
\text { AZD5423 } 250 \mu \mathrm{g}: \\
\text { IV, AZD5423 } \\
\text { I } 200 \mu \mathrm{g}: \text { PO }\end{array}$ \\
\hline $\begin{array}{l}\text { Melin et al, } \\
2017^{48,49}\end{array}$ & $\begin{array}{l}\text { NCT0I635985, } \\
\text { Study B }\end{array}$ & $\begin{array}{l}\text { Phase I, single-center, } \\
\text { partly randomized, } \\
\text { open-label, 6-way, } \\
\text { crossover }\end{array}$ & I & 18 & $\begin{array}{l}\text { AZD5423 } 250 \mu \mathrm{g}, \\
332 \mu \mathrm{g}, 405 \mu \mathrm{g}, 456 \\
\mu \mathrm{g}, 523 \mu \mathrm{g}, 1200 \mu \mathrm{g} \\
\text { (single dose) }\end{array}$ & NA & $\begin{array}{l}\text { AZD5423 } 332 \mu \mathrm{g}, \\
405 \mu \mathrm{g}, 456 \mu \mathrm{g}, \\
523 \mu \mathrm{g}: \text { oral } \\
\text { inhalation, AZD5423 } \\
250 \mu \mathrm{g}: \text { IV, AZD5423 } \\
1200 \mu \mathrm{g}: \text { PO }\end{array}$ \\
\hline $\begin{array}{l}\text { Chen et al, } \\
2017^{51,52}\end{array}$ & NCT02648438 & $\begin{array}{l}\text { Phase I, single-center, } \\
\text { partiy randomized, } \\
\text { open-label, 4-period, } \\
\text { parallel }\end{array}$ & I & 30 & $\begin{array}{l}\text { AZD7594 I50 } \mu \mathrm{g}, \\
400 \mu \mathrm{g}, 1200 \mu \mathrm{g}\end{array}$ & NA & $\begin{array}{l}\text { AZD7594 } 400 \mu \mathrm{g}: \\
\text { oral inhalation, } \\
\text { AZD7594 I50 } \mu \mathrm{g}: \\
\text { IV, AZD7594 } \\
\text { I } 200 \mu \mathrm{g}: \text { PO }\end{array}$ \\
\hline $\begin{array}{l}\text { Werkström } \\
\text { et al, } 2016^{50}\end{array}$ & NA, Study A & $\begin{array}{l}\text { Single-dose Phase I, } \\
\text { single-center, } \\
\text { randomized, double- } \\
\text { blind, placebo- } \\
\text { controlled, escalated- } \\
\text { dose }\end{array}$ & I & 59 & $\begin{array}{l}\text { AZD5423 8.4-2400 } \\
\mu g\end{array}$ & Placebo & Oral inhalation \\
\hline $\begin{array}{l}\text { Werkström } \\
\text { et al, } 2016^{50}\end{array}$ & NA, Study B & $\begin{array}{l}\text { Multiple-ascending dose } \\
\text { Phase I, randomized, } \\
\text { double-blind, placebo- } \\
\text { controlled, parallel }\end{array}$ & 14 & 27 & $\begin{array}{l}\text { AZD5423 I25 } \mu \mathrm{g} \\
\text { (q.d.), } 375 \mu \mathrm{g} \text { (q.d.), } \\
499 \mu \mathrm{g} \text { (q.d.) }\end{array}$ & Placebo & Oral inhalation \\
\hline $\begin{array}{l}\text { Werkström } \\
\text { et al, } 2016^{50}\end{array}$ & NA, Study C & $\begin{array}{l}\text { Single- and multiple- } \\
\text { ascending dose Phase I, } \\
\text { single-center, } \\
\text { randomized, double- } \\
\text { blind, placebo- } \\
\text { controlled, parallel }\end{array}$ & 14 & 30 & $\begin{array}{l}\text { AZD5423 } 50 \mu \mathrm{g} \\
\text { (single-dose), } 100 \\
\mu \mathrm{g} \text { (q.d.), } 300 \mu \mathrm{g} \text { (q. } \\
\text { d.) }\end{array}$ & Placebo & Oral inhalation \\
\hline
\end{tabular}




\begin{tabular}{|c|c|c|c|c|c|c|c|c|c|}
\hline $\begin{array}{l}\text { Inhaler } \\
\text { Device }\end{array}$ & $\begin{array}{l}\text { Patient } \\
\text { Characteristics }\end{array}$ & $\begin{array}{l}\text { Age } \\
\text { (Years) }\end{array}$ & $\begin{array}{l}\text { Male } \\
(\%)\end{array}$ & $\begin{array}{l}\text { Current } \\
\text { Smokers } \\
(\%)\end{array}$ & $\begin{array}{l}\text { Smoking } \\
\text { History } \\
\text { (Pack- } \\
\text { Years) }\end{array}$ & $\begin{array}{l}\text { BMI (kgl } \\
\left.\mathbf{m}^{2}\right)\end{array}$ & $\begin{array}{l}\text { Pre- } \\
\text { Bronchodilator } \\
\text { FEV, Predicted } \\
\text { (\%) }\end{array}$ & $\begin{array}{l}\text { Investigated } \\
\text { Outcomes }\end{array}$ & $\begin{array}{l}\text { Jadad } \\
\text { Score }\end{array}$ \\
\hline DPI & $\begin{array}{l}\text { Mild to moderate } \\
\text { asthmatic (pre- } \\
\text { bronchodilator } \mathrm{FEV}_{\text {, }} \\
\geq 40 \% \text { and } \leq 90 \% \text { of } \\
\text { predicted, } \mathrm{F}_{\mathrm{E}} \mathrm{NO} \geq 25 \\
\mathrm{Ppb} \text { ) }\end{array}$ & 50.8 & 82.9 & 0.0 & NA & 27.3 & $\geq 40.0$ and $\leq 90.0$ & $\begin{array}{l}\text { Lung function, } \\
\text { symptom } \\
\text { control, PK, } \\
\text { PD, and safety }\end{array}$ & 5 \\
\hline DPI & $\begin{array}{l}\text { Healthy Japanese } \\
\text { subjects (NA) }\end{array}$ & 34.0 & 100.0 & NA & NA & 23.1 & NA & $\begin{array}{l}\text { PK, PD, and } \\
\text { safety }\end{array}$ & 3 \\
\hline $\begin{array}{l}\text { Jet nebulizer, } \\
\text { hand-held } \\
\text { vibrating mesh } \\
\text { nebulizer }\end{array}$ & $\begin{array}{l}\text { Healthy subjects (NA) } \\
\text { and mild allergic } \\
\text { asthmatic (pre- } \\
\text { bronchodilator FEV } \\
\geq 70 \% \text { ) }\end{array}$ & $18.0-42.0$ & 100.0 & 0.0 & NA & $20.5-28.4$ & $\geq 70.0$ & PK and safety & I \\
\hline $\begin{array}{l}\text { Jet nebulizer, } \\
\text { hand-held } \\
\text { vibrating mesh } \\
\text { nebulizer, } \\
\text { approved DPI, } \\
\text { new DPI }\end{array}$ & Healthy subjects (NA) & $21.0-40.0$ & 100.0 & 0.0 & NA & $19.2-30.0$ & NA & PK and safety & I \\
\hline $\begin{array}{l}\text { Mono-dose } \\
\text { DPI, MDI }\end{array}$ & Healthy subjects (NA) & 32.9 & 100.0 & 0.0 & NA & $18.0-30.0$ & NA & PK and safety & 1 \\
\hline Jet nebulizer & Healthy subjects (NA) & 26.0 & 100.0 & NA & NA & 24.0 & NA & $\begin{array}{l}\mathrm{PK}, \mathrm{PD} \text {, and } \\
\text { safety }\end{array}$ & 2 \\
\hline Jet nebulizer & Healthy subjects (NA) & 28.0 & 100.0 & NA & NA & 25.0 & NA & $\begin{array}{l}\mathrm{PK}, \mathrm{PD} \text {, and } \\
\text { safety }\end{array}$ & 2 \\
\hline $\begin{array}{l}\text { Hand-held } \\
\text { vibrating mesh } \\
\text { nebulizer }\end{array}$ & $\begin{array}{l}\text { Healthy Japanese } \\
\text { subjects (NA) }\end{array}$ & 29.6 & 100.0 & NA & NA & 22.2 & NA & $\begin{array}{l}\mathrm{PK}, \mathrm{PD} \text {, and } \\
\text { safety }\end{array}$ & 2 \\
\hline
\end{tabular}

(Continued) 
Table I (Continued).

\begin{tabular}{|c|c|c|c|c|c|c|c|}
\hline $\begin{array}{l}\text { Study and } \\
\text { Year }\end{array}$ & $\begin{array}{l}\text { ClinicalTrials. } \\
\text { gov Identifier } \\
\text { and/or } \\
\text { Company ID }\end{array}$ & $\begin{array}{l}\text { Study } \\
\text { Characteristics }\end{array}$ & $\begin{array}{l}\text { Treatment } \\
\text { Duration } \\
\text { (Days) }\end{array}$ & $\begin{array}{l}\text { Number } \\
\text { of } \\
\text { Analyzed } \\
\text { Patients }\end{array}$ & $\begin{array}{l}\text { Drugs, Doses, } \\
\text { and Regimen of } \\
\text { Administration }\end{array}$ & Comparator & $\begin{array}{l}\text { Route of } \\
\text { Administration }\end{array}$ \\
\hline $\begin{array}{l}\text { Leaker et al, } \\
2015^{53}\end{array}$ & $\begin{array}{l}\text { NСT00483899, } \\
\text { Part B }\end{array}$ & $\begin{array}{l}\text { Phase II, multicenter, } \\
\text { randomized, double- } \\
\text { blind, placebo- } \\
\text { controlled, 3-way, } \\
\text { crossover }\end{array}$ & 21 & 21 & $\begin{array}{l}\text { GW870086X I mg } \\
\text { (q.d.), } 3 \mathrm{mg} \text { (q.d.) }\end{array}$ & Placebo & Oral inhalation \\
\hline $\begin{array}{l}\text { Gauvreau et } \\
\text { al, } 2015^{54,55}\end{array}$ & NCTOI 225549 & $\begin{array}{l}\text { Phase II, multicenter, } \\
\text { randomized, double- } \\
\text { blind, double-dummy, } \\
\text { placebo- and active- } \\
\text { controlled, 4-way, } \\
\text { crossover }\end{array}$ & 7 & 20 & $\begin{array}{l}\text { AZD5423 } 75 \mu g \text { (q. } \\
\text { d.), } 300 \mu g \text { (q.d.), } \\
\text { and BUD } 200 \mu g \text { (b. } \\
\text { i.d.) }\end{array}$ & Placebo & Oral inhalation \\
\hline $\begin{array}{l}\text { Bareille et } \\
\text { al, } 2013^{56,57}\end{array}$ & NCT00945932 & $\begin{array}{l}\text { Phase II, multicenter, } \\
\text { randomized, double- } \\
\text { blind, placebo- } \\
\text { controlled, 2-way } \\
\text { crossover }\end{array}$ & 28 & 36 & $\begin{array}{l}\text { GW870086X I mg } \\
\text { (q.d.) }\end{array}$ & Placebo & Oral inhalation \\
\hline $\begin{array}{l}\text { Allen et al, } \\
2013^{58,59}\end{array}$ & $\begin{array}{l}\text { NCT0II60003, } \\
\text { SIGII3209 }\end{array}$ & $\begin{array}{l}\text { Dose-ascending, Phase } \\
\text { I, single-center, } \\
\text { randomized, double- } \\
\text { blind, placebo- } \\
\text { controlled, 3-way } \\
\text { crossover }\end{array}$ & 14 & 12 & $\begin{array}{l}\text { GW870086X } 5 \mathrm{mg} \\
\text { (q.d.), } 8.75 \mathrm{mg} \text { (q.d.) }\end{array}$ & Placebo & Oral inhalation \\
\hline $\begin{array}{l}\text { Bareille } \\
\text { et al, } \\
2013^{60,63}\end{array}$ & $\begin{array}{l}\text { NCT0I245426, } \\
\text { SIGII } 4749\end{array}$ & $\begin{array}{l}\text { Phase II, multicenter, } \\
\text { randomized, double- } \\
\text { blind, placebo- } \\
\text { controlled, parallel }\end{array}$ & 27 & 135 & $\begin{array}{l}\text { GW870086X } 2 \text { mg } \\
\text { (q.d.), } 4 \text { mg (q.d.), } \\
3 \text { mg (q.d. added at } \\
\text { interim) }\end{array}$ & Placebo & Oral inhalation \\
\hline $\begin{array}{l}\text { Bareille } \\
\text { et al, } \\
2013^{61,64}\end{array}$ & $\begin{array}{l}\text { NCT00857857, } \\
\text { SIGII } 0762\end{array}$ & $\begin{array}{l}\text { Phase lla, multicenter, } \\
\text { randomized, double- } \\
\text { blind, placebo- and } \\
\text { active-controlled, } \\
\text { incomplete block, 3-way } \\
\text { crossover }\end{array}$ & 13 & 24 & $\begin{array}{l}\text { GW870086X } \\
0.25 \mathrm{mg} \text { (q.d.), I mg } \\
\text { (q.d.), } 3 \mathrm{mg} \text { (q.d.), } \\
\text { and FP } 0.25 \mathrm{mg} \text { (b.i. } \\
\text { d.) }\end{array}$ & Placebo & Oral inhalation \\
\hline $\begin{array}{l}\text { Boulet et al, } \\
2009^{62}\end{array}$ & NA & $\begin{array}{l}\text { Dose-ascending, Phase } \\
\text { I, multicenter, } \\
\text { randomized, double- } \\
\text { blind, escalated-dose }\end{array}$ & 21 & 26 & $\begin{array}{l}\text { TPI I020 } 600 \mu \mathrm{g} \text { (b. } \\
\text { i.d. for } 2 \text { weeks), } \\
\text { I } 200 \mu \mathrm{g} \text { (b.i.d. for I } \\
\text { week), BUD } 400 \mu \mathrm{g} \\
\text { (b.i.d. for } 2 \text { weeks), } \\
800 \mu \mathrm{g} \text { (b.i.d. for I } \\
\text { week) }\end{array}$ & NA & Oral inhalation \\
\hline
\end{tabular}

Abbreviations: b.i.d., twice daily; BMI, body mass index; BUD, budesonide; DPI, dry powder inhaler; $\mathrm{F}_{\mathrm{E}} \mathrm{NO}$, fraction exhaled of nitric oxide; $\mathrm{FEV}$, forced expiratory volume in I second; FP, fluticasone propionate; IV, intravenous administration; MDI, metered-dose inhaler; NA, not available; PD, pharmacodynamics; PMDI, pressurized metered-dose inhaler; PO, oral administration; PK, pharmacokinetics; ppb, parts per billion; q.d., once daily. 


\begin{tabular}{|c|c|c|c|c|c|c|c|c|c|}
\hline $\begin{array}{l}\text { Inhaler } \\
\text { Device }\end{array}$ & $\begin{array}{l}\text { Patient } \\
\text { Characteristics }\end{array}$ & $\begin{array}{l}\text { Age } \\
\text { (Years) }\end{array}$ & $\begin{array}{l}\text { Male } \\
(\%)\end{array}$ & $\begin{array}{l}\text { Current } \\
\text { Smokers } \\
(\%)\end{array}$ & $\begin{array}{l}\text { Smoking } \\
\text { History } \\
\text { (Pack- } \\
\text { Years) }\end{array}$ & $\begin{array}{l}\text { BMI (kgl } \\
\left.\mathrm{m}^{2}\right)\end{array}$ & $\begin{array}{l}\text { Pre- } \\
\text { Bronchodilator } \\
\text { FEV, Predicted } \\
\text { (\%) }\end{array}$ & $\begin{array}{l}\text { Investigated } \\
\text { Outcomes }\end{array}$ & $\begin{array}{l}\text { Jadad } \\
\text { Score }\end{array}$ \\
\hline DPI & $\begin{array}{l}\text { Mild asthmatic (pre- } \\
\text { bronchodilator FEV, } \\
\geq 60 \% \text { of predicted, } \\
\mathrm{F}_{\mathrm{E}} \mathrm{NO} \geq 25 \mathrm{ppb} \text { ) }\end{array}$ & 28.0 & 100.0 & NA & NA & 24.0 & 87.0 & $\begin{array}{l}\text { Lung function, } \\
\text { PK, PD, and } \\
\text { safety }\end{array}$ & 3 \\
\hline $\begin{array}{l}\text { AZD5423: } \\
\text { vibrating mesh } \\
\text { nebulizer I- } \\
\text { neb }^{\circledR} \text {, BUD: } \\
\text { DPI } \\
\left(\text { Turbuhaler }^{\circledR}\right)\end{array}$ & $\begin{array}{l}\text { Mild atopic asthmatic } \\
\text { (pre-bronchodilator } \\
\mathrm{FEV}_{1} \geq 70 \% \text { of } \\
\text { predicted, NA) }\end{array}$ & 29.6 & 55.0 & 0.0 & NA & NA & 92.1 & $\begin{array}{l}\text { Lung function, } \\
\text { PD, and safety }\end{array}$ & 4 \\
\hline $\begin{array}{l}\text { DPI } \\
\left.\text { (Diskhaler }^{\circledR}\right)\end{array}$ & $\begin{array}{l}\text { Mild to moderate } \\
\text { asthmatic (pre- } \\
\text { bronchodilator FEV } \\
\geq 40 \% \text { and } \leq 85 \% \text { of } \\
\text { predicted) }\end{array}$ & 49.0 & 56.0 & 0.0 & $\leq 10.0$ & 26.0 & 73.7 & $\begin{array}{l}\text { Lung function, } \\
\text { and safety }\end{array}$ & 4 \\
\hline $\begin{array}{l}\text { Nebulizer } \\
\text { (NA) }\end{array}$ & Healthy subjects (NA) & 54.0 & 100.0 & 0.0 & $>5.0$ & 24.5 & NA & $\begin{array}{l}\text { PK, PD, and } \\
\text { safety }\end{array}$ & 3 \\
\hline NA & $\begin{array}{l}\text { Persistent asthmatic } \\
\text { (pre-bronchodilator } \\
\mathrm{FEV}, \geq 60 \% \text { and }<85 \% \\
\text { of predicted) }\end{array}$ & 43.5 & 80.7 & 0.0 & $\leq 10.0$ & 25.4 & NA & $\begin{array}{l}\text { Lung function, } \\
\text { and safety }\end{array}$ & 5 \\
\hline DPI & $\begin{array}{l}\text { Mild asthmatic (pre- } \\
\text { bronchodilator FEV } \\
>65 \% \text { of predicted) }\end{array}$ & 39.4 & 100.0 & 0.0 & $\leq 10.0$ & NA & $>65.0$ & $\begin{array}{l}\text { Lung function, } \\
\text { PD, and safety }\end{array}$ & 5 \\
\hline DPI & $\begin{array}{l}\text { Mild asthmatic (pre- } \\
\text { bronchodilator FEV } \\
\geq 75 \% \text { of predicted) }\end{array}$ & 34.4 & 51.9 & 100.0 & 10.8 & NA & 85.3 & $\begin{array}{l}\mathrm{PK}, \mathrm{PD} \text {, and } \\
\text { safety }\end{array}$ & 3 \\
\hline
\end{tabular}


Bias arising from the randomization process Bias due to deviations from intended interventions Bias due to missing outcome data Bias in measurement of the outcome Bias in selection of the reported result Overall risk of bias

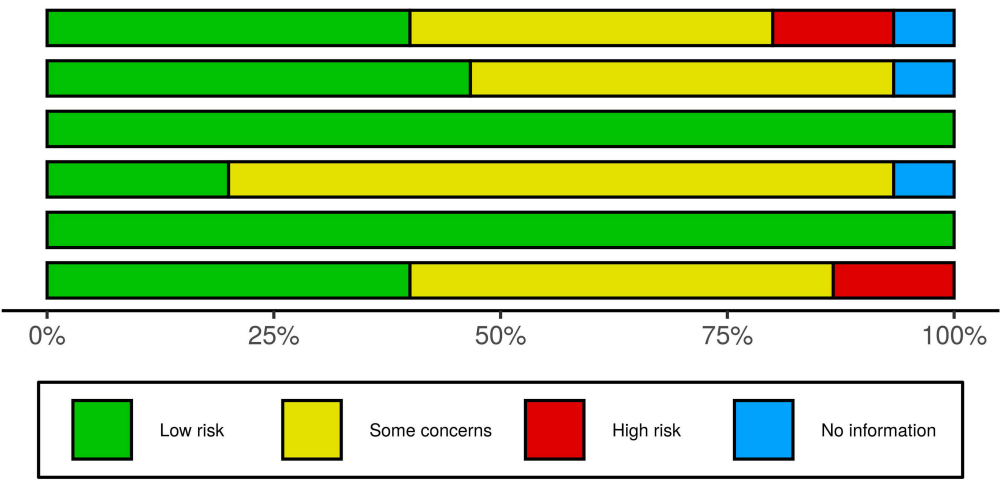

Figure 3 Weighted plot for the assessment of the overall risk of bias via the Cochrane RoB 2 tool ( $n=15$ RCTs). Abbreviation: RCT, randomized controlled trial.

values of $3.7-14.4 \mathrm{nmol} / \mathrm{L}{ }^{50}$ The $t_{\max }$ values were similar across the investigated doses. ${ }^{50}$ In the multiple-ascending dose study, AZD5423 $499 \mu \mathrm{g}$ induced a partial suppression of plasma cortisol and a marked reduction in urinary excreted cortisol level. ${ }^{50}$

In an open-label partly randomized Phase I study (NCT01310322) performed in healthy subjects and mildly asthmatic patients, AZD5423 420-450 $\mu \mathrm{g}$ administered via a hand-held vibrating mesh nebulizer and a jet nebulizer achieved a pulmonary bioavailability of $27.0-49.0 \%$, an oral bioavailability of $1.3-2.1 \%$, a $C_{\max }$ of $3.29-7.90 \mathrm{nmol} / \mathrm{L}$, a $t_{\max }$ of $0.17 \mathrm{~h}$, and a half-life $\left(t_{1 / 2}\right)$ of $11.5-21.4 \mathrm{~h}$. Intravenous (IV) AZD5423 $250 \mu \mathrm{g}$ produced a $C_{\max }$ of $0.6 \mathrm{nmol} / \mathrm{L}$, a $t_{\max }$ of $0.42 \mathrm{~h}$, and a $t_{1 / 2}$ of $25.0 \mathrm{~h}^{47,48}$ Oral AZD5423 $1200 \mu \mathrm{g}$ elicited a $C_{\max }$ of $0.841 \mathrm{nmol} / \mathrm{L}$, a $t_{\max }$ of $0.84 \mathrm{~h}$, and a $t_{1 / 2}$ of $4.0 \mathrm{~h} .^{47,48}$ Drug disposition showed a multi-phasic profile characterized by a fast process of absorption from the alveolar space into the systemic circulation due to the small particle size of the nebulized formulations. ${ }^{4,48}$

In an open-label partly randomized Phase I study (NCT01635985) performed in healthy subjects, the pulmonary bioavailability of AZD5423 405-420 $\mu \mathrm{g}$ via a hand-held vibrating mesh nebulizer was $27.0 \%$, while it was 37.0 $49.0 \%$ for AZD5423 450-523 $\mu \mathrm{g}$ via a jet nebulizer, $31.0 \%$ for AZD5423 $456 \mu \mathrm{g}$ via the approved dry powder inhaler (DPI) Turbuhaler ${ }^{\circledR}$, and $46.0 \%$ for AZD5423 $332 \mu \mathrm{g}$ via a new DPI. ${ }^{48,49}$ The oral bioavailability of AZD5423 1200 $\mu \mathrm{g}$ was low (2.3-3.4\%). ${ }^{48,49}$ The $C_{\max }$ of AZD5423 administered via inhalation was $2.36-4.04 \mathrm{nmol} / \mathrm{L}, t_{\max }$ was $0.17-$ $0.33 \mathrm{~h}$, and $t_{1 / 2} 17.9-26.6 \mathrm{~h}^{48,49}$ AZD5423 via DPIs was absorbed more slowly than AZD5423 administered via nebulized formulations due to the different particle size. ${ }^{48,49}$ The slow absorption of AZD5423 via the approved DPI and the new DPI was permeation rate limited, and related to a relatively short retention time in the lung. ${ }^{48,49}$

AZD5423 was generally well tolerated and neither SAEs nor clinically relevant alterations were reported in the studies. AZD5423 is not currently under investigation for asthma; the last update was posted in 2015 and the status of the clinical trials remains to be completed.

Overall, AZD5423 exerted a superior efficacy compared to DEX and BUD in vitro. ${ }^{39}$ AZD5423 showed a doseproportional plasma exposure, limited accumulation, and a dose-related effect on plasma and urine cortisol in healthy subjects, ${ }^{50}$ whereas it effectively reduced the allergeninduced response in mild allergic asthma. ${ }^{54,55}$ The pulmonary bioavailability was related to the device and considerably lower than that predicted in vitro. ${ }^{47,48}$ AZD5423 is retained in the lung for a short time and its oral bioavailability is low. $^{48,49}$ AZD5423 was generally well tolerated. ${ }^{48,49,54,55}$

\section{AZD7594}

The SEGRA AZD7594 (3-[5-[(1R,2S)-2-(2,2-difluoropropanoylamino)-1-(2,3-dihydro-1,4-benzodioxin-6-yl)propoxy]indazol-1-yl]-N-[(3R)-oxolan-3-yl]benzamide) has recently entered clinical development for the treatment of asthma.

AZD7594 is a potent and selective agonist of the progesterone, androgen, mineralocorticoid, and estrogen$\alpha$ and $\beta$ receptors. In human alveolar macrophages stimulated by lipopolysaccharide (LPS), AZD7594 potently reduced the release of tumor necrosis factor-alpha $($ TNF- $\alpha){ }^{39}$ AZD7594 via DPI inhibited in a dosedependent manner the lung edema induced in a crosslinked dextran gel rat model of asthma, showing an improved therapeutic ratio and prolonged anti- 


\begin{tabular}{|c|c|c|c|c|c|c|c|c|}
\hline$\underbrace{2}_{2}$ & 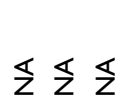 & 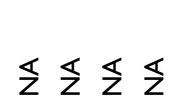 & $\S$ & \multicolumn{3}{|c|}{ 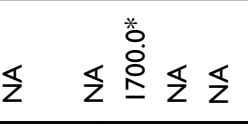 } & 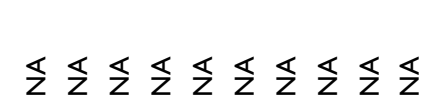 & $\S$ \\
\hline ป & 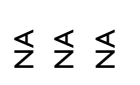 & 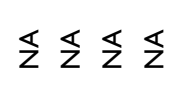 & $\S$ & 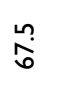 & \multicolumn{2}{|c|}{$\stackrel{0}{a} \underset{\sigma}{\stackrel{f}{Z}} \overline{\dot{m}}$} & 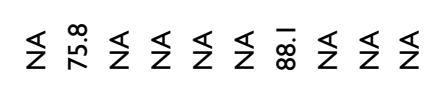 & $\S$ \\
\hline 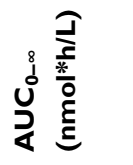 & 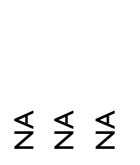 & 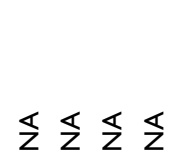 & 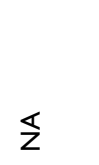 & $\S$ & \multicolumn{2}{|c|}{ 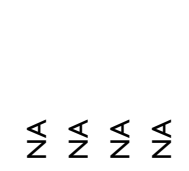 } & 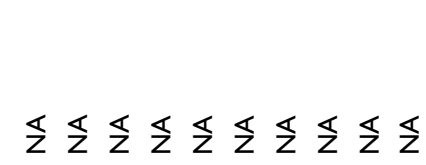 & $\S$ \\
\hline 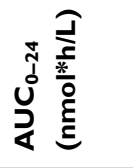 & 过 $\mathbb{z}$ & $\hat{0} \mathbb{z} \stackrel{\mathbb{N}}{z}$ & $\S$ & $\stackrel{\text { 系 }}{+}$ & \multicolumn{2}{|c|}{ 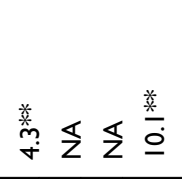 } & 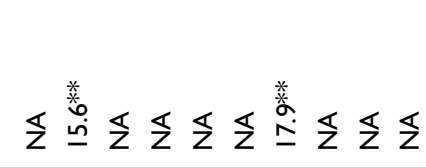 & $\S$ \\
\hline 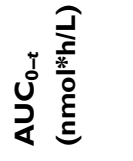 & 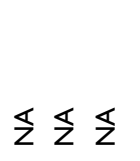 & 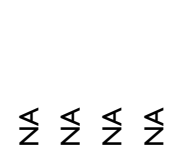 & 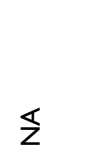 & $\S$ & \multicolumn{2}{|c|}{$\mathbb{z} \stackrel{+}{0} \stackrel{\infty}{=} \mathbb{Z}$} & 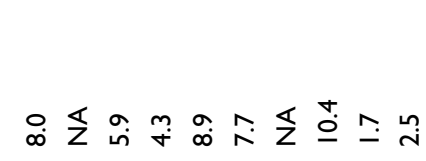 & $\S$ \\
\hline د د & 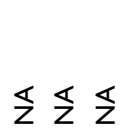 & 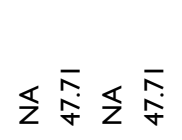 & $\underset{\hat{q}}{\bar{q}}$ & $\S$ & \multicolumn{2}{|c|}{ 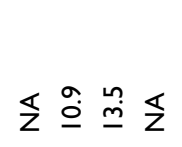 } & 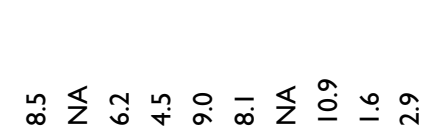 & 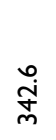 \\
\hline$\cong$ & 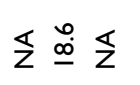 & 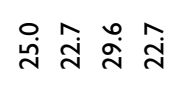 & $\hat{\mathrm{N}}$ & 莣 & \multicolumn{2}{|c|}{ 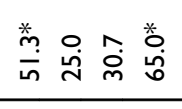 } & 屰 & $\S$ \\
\hline$\stackrel{x}{\underline{E}}$ & $\stackrel{\infty}{0} \stackrel{\sim}{\sim} \stackrel{n}{\sim}$ & 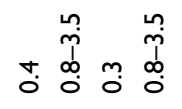 & $\underset{\substack{n \\
0 \\
\infty \\
0 \\
0}}{0}$ & $\overline{0}$ & \multicolumn{2}{|c|}{$\stackrel{\infty}{0} \stackrel{\circ}{0}$ : } & 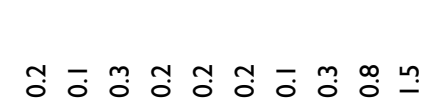 & $\stackrel{\circ}{m}$ \\
\hline है & 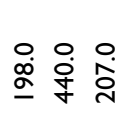 & 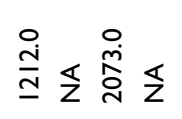 & $\S$ & $\frac{\hat{A}}{\frac{\bar{d}}{\hat{N}}}$ & \multicolumn{2}{|c|}{ 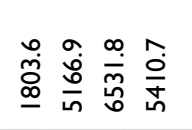 } & 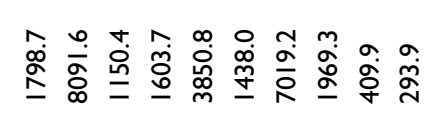 & กู \\
\hline 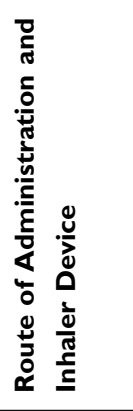 & \multicolumn{3}{|c|}{ 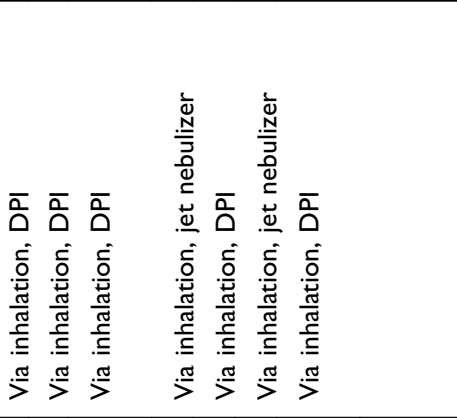 } & \multicolumn{4}{|c|}{ 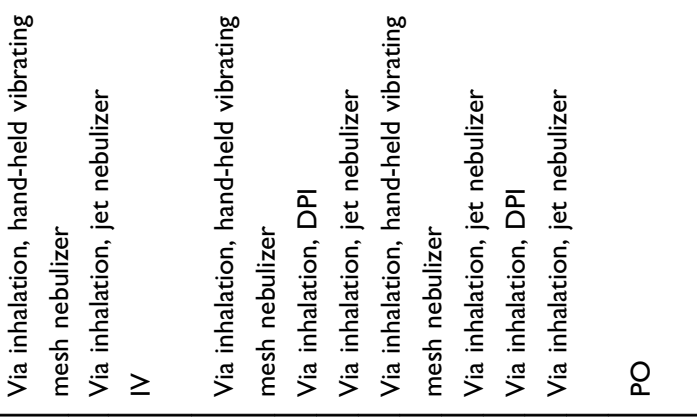 } & 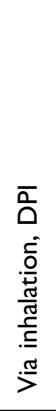 \\
\hline 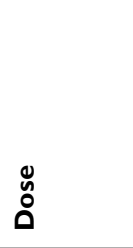 & 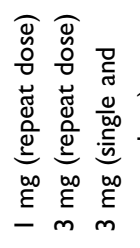 & 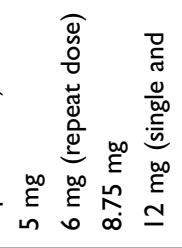 & 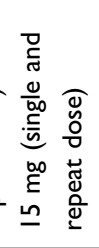 & 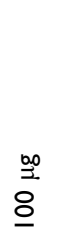 & 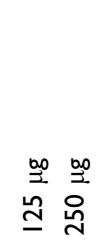 & 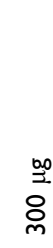 & 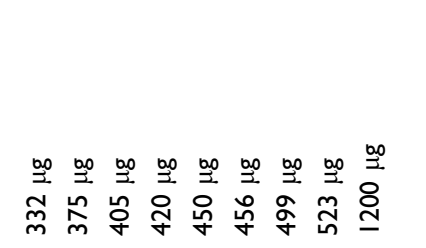 & $\begin{array}{l}\text { no } \\
\stackrel{2}{0} \\
8\end{array}$ \\
\hline 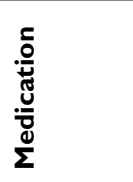 & 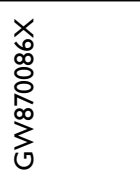 & & & 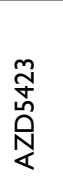 & & & & $\frac{\stackrel{\circ}{\grave{O}}}{\bar{\alpha}}$ \\
\hline
\end{tabular}




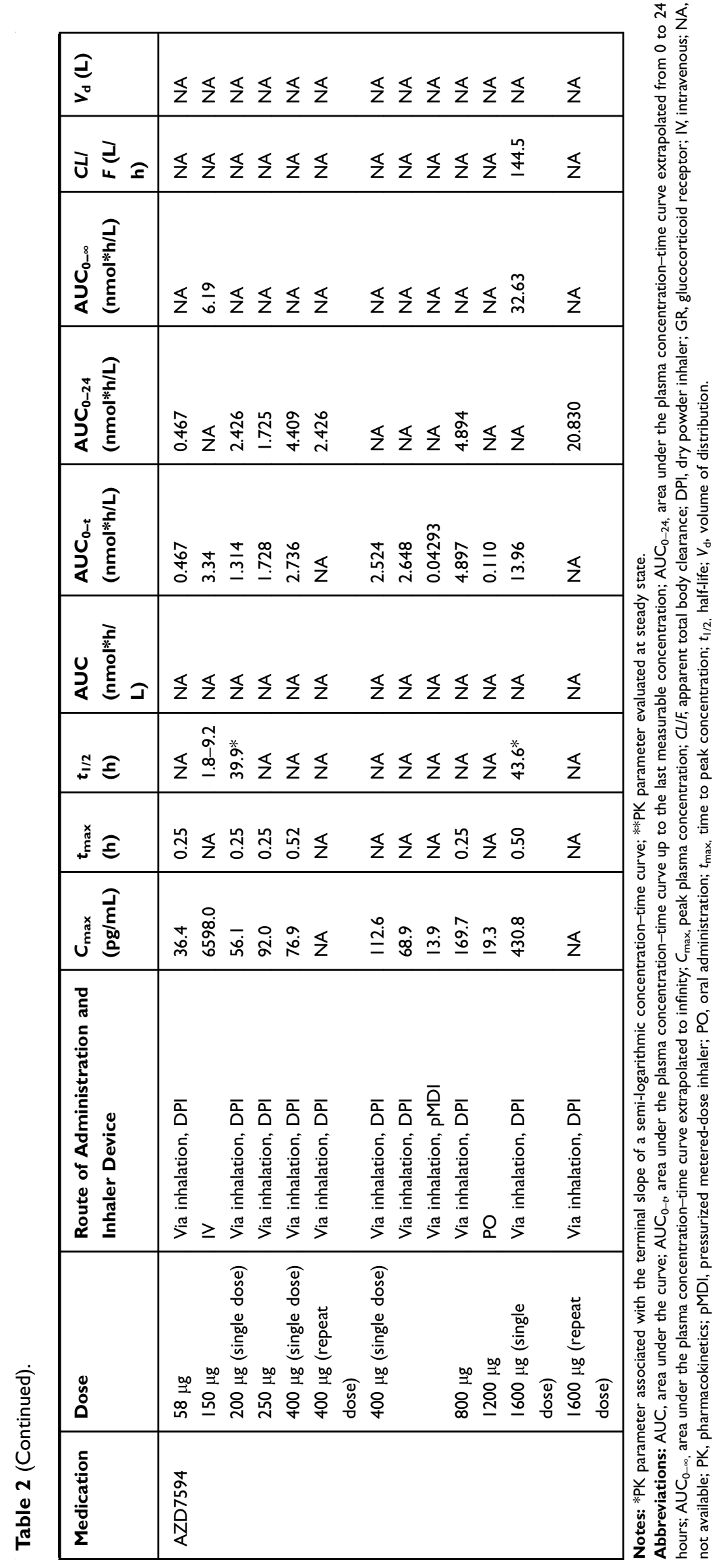




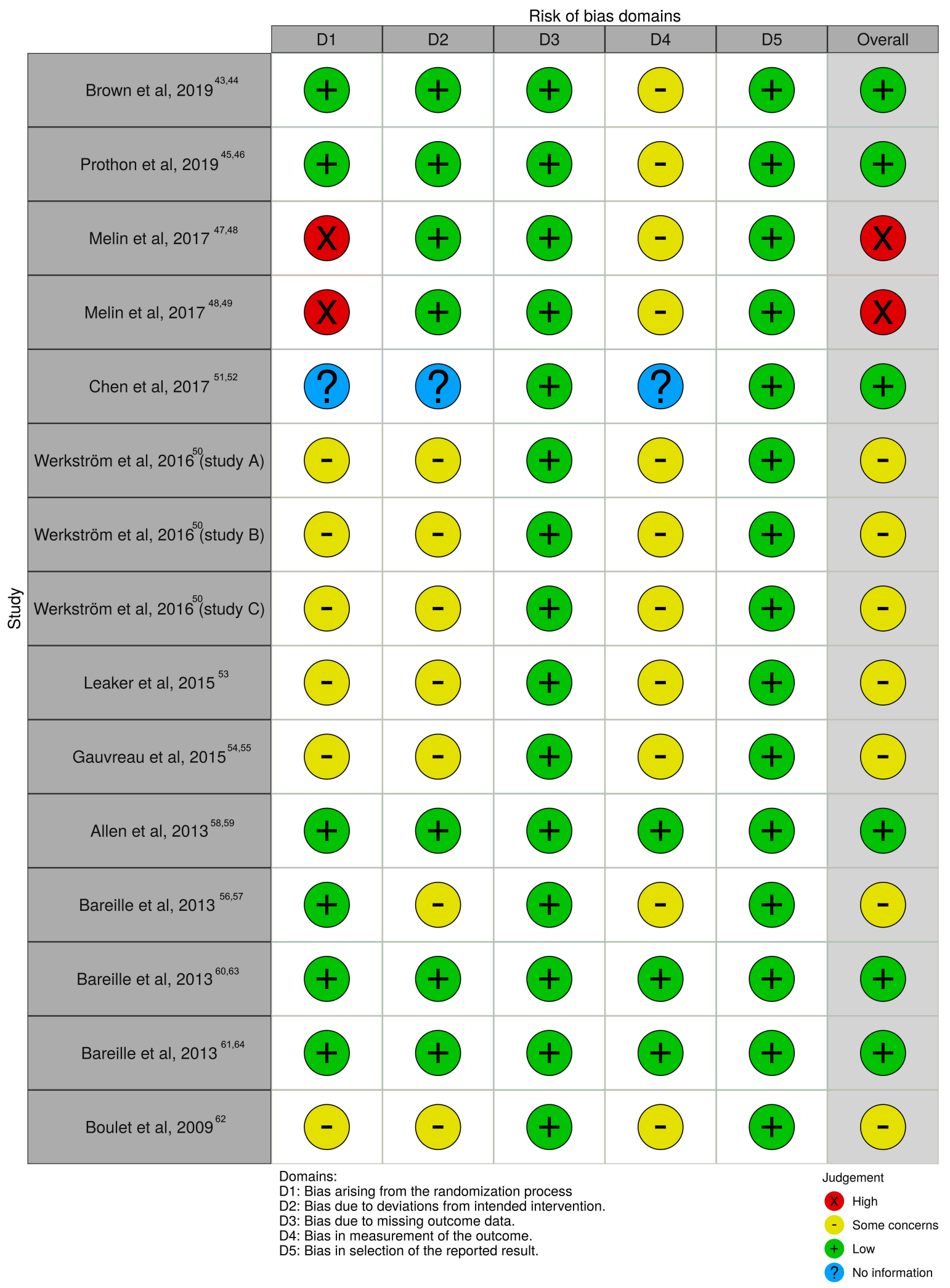

Figure 4 Traffic light plot for assessing the specific risk of bias of each RCT via the Cochrane RoB 2 tool. DI: bias arising from the randomization process; D2: bias due to deviations from intended intervention; D3: bias due to missing outcome data; D4: bias in measurement of the outcome; D5: bias in selection of the reported result. Red circle indicates high risk of bias, yellow circle indicates some concerns on the risk of bias, green circle represents low risk of bias, and blue circle refers to lack of information due to limited data results available on Clinicaltrials.gov database.

Abbreviations: RCT, randomized controlled trial; RoB, Risk of Bias. 
inflammatory activity compared to fluticasone propionate (FP). ${ }^{39}$ In a murine model of LPS- or tobacco smokeinduced pulmonary inflammation, the intra-tracheal administration of AZD7594 dose-dependently reduced lung neutrophilia. ${ }^{39}$

A Phase I RCT (NCT02645253) investigated the PK and PD of single and repeat doses of AZD7594 200-1600 $\mu \mathrm{g}$ delivered via a DPI in healthy Japanese male subjects. ${ }^{45,46}$ Plasma exposure indicated dose-proportional PK. ${ }^{4,46}$ The $C_{\text {max }}$ of single doses of AZD7594 200-1600 $\mu \mathrm{g}$ was 56.05$430.8 \mathrm{pmol} / \mathrm{L}$ and $t_{\max }$ was $0.25-0.50 \mathrm{~h} .^{45,46}$ Only in a few subjects, the terminal $t_{1 / 2}$ was reliably estimated to be $40.0-44.0 \mathrm{~h}$ since the investigated drug showed a slow terminal phase and achieved low sustained concentrations over the sampling period. ${ }^{45,46}$ Urinary excretion of AZD7594 was considered a negligible elimination pathway since less than $0.02 \%$ of unchanged drug was excreted in the urine. ${ }^{45,46}$ Only multiple doses of AZD7594 $1600 \mu \mathrm{g}$ elicited a partial cortisol suppression compared to placebo, although no reduction in osteocalcin level occurred at any dose. ${ }^{45,46}$

AZD7594 58-800 $\mu \mathrm{g}$ induced a rapid increase in plasma concentrations with a mean $C_{\max }$ of 36.4-169.7 $\mathrm{pmol} / \mathrm{L}$, followed by a rapid decline in plasma concentration and a slow elimination phase. ${ }^{4,44}$ The $t_{\max }$ was $0.25 \mathrm{~h}$ at all doses. ${ }^{43,44}$

A Phase I device bridging trial (NCT02648438) assessed that when administered to healthy male subjects, AZD7594 $400 \mu \mathrm{g}$ via mono- and multi-dose DPIs produced a systemic bioavailability of $28.2-30.9 \%$. A single dose of AZD7594 $400 \mu \mathrm{g}$ via a pressurized metered-dose inhaler (pMDI) induced a low systemic exposure. ${ }^{51,52}$ AZD7594 $400 \mu \mathrm{g}$ via a mono-dose DPI induced a slow absorption with a $C_{\max }$ of $112.6 \mathrm{pmol} / \mathrm{L}$, compared to multi-dose DPI and pMDI, which achieved $C_{\max }$ values of $68.88 \mathrm{pmol} / \mathrm{L}$ and $13.93 \mathrm{pmol} / \mathrm{L}$, respectively. ${ }^{51,52}$ AZD7594 IV and PO produced $C_{\max }$ values of $6598 \mathrm{pmol} / \mathrm{L}$ and $19.31 \mathrm{pmol} / \mathrm{L}$, respectively. ${ }^{51,52}$ Following an IV single dose of AZD7594 $150 \mu \mathrm{g}$, the elimination $t_{1 / 2}$ was $1.8-9.2 \mathrm{~h}$, while after inhalation this value was considerably longer. ${ }^{51,52}$

In a Phase IIa RCT (NCT02479412) performed in patients with mild to moderate asthma, once-daily AZD7594 $800 \mu \mathrm{g}$ via a DPI, but not 58-250 $\mu \mathrm{g}$, improved trough $\mathrm{FEV}_{1}$ compared to placebo (mean difference [MD] $148 \mathrm{~mL}){ }^{43,44}$ While evening PEF improved at all the investigated doses compared to placebo, morning PEF improved only at the highest dose. $^{43,44}$ At all doses, AZD7594 induced a reduction in $\mathrm{F}_{\mathrm{E}} \mathrm{NO}$, and when administered at 250-800 $\mu \mathrm{g}$, it decreased the daily use of rescue medication, compared to placebo ( $\mathrm{MD}-0.49$ to -0.80 inhalations/day). ${ }^{43,44}$ Asthma control markedly improved with AZD7594 58-800 $\mu \mathrm{g}$, but only AZD7594 $800 \mu \mathrm{g}$ increased the number of symptom-free days and asthma control days and reduced night-time awakenings, compared to placebo. ${ }^{43,44}$ No difference in plasma cortisol level was observed between AZD7594 and placebo. ${ }^{43,44}$

AZD7594 was safe and well tolerated, and no SAEs were reported in the studies. ${ }^{43-46,51,52}$ AZD7594 is still under investigation for asthma in an ongoing Phase I study. ${ }^{67}$

In general, inhaled AZD7594 exerted potent antiinflammatory effects in animal models of pulmonary inflammation. ${ }^{39}$ AZD7594 improved lung function, asthma control, and symptoms, and reduced airway inflammation in mild to moderate asthma. ${ }^{43,44}$ AZD7594 showed a doseproportional plasma exposure and moderate accumulation, with a marginal impact on systemic markers of GC activity in healthy subjects. ${ }^{45,46}$ The PK characteristics AZD7594 support the once-daily dosing. ${ }^{51,52}$ AZD7594 was characterized by a favorable safety profile. ${ }^{43,44}$

\section{Compound 7}

Compound 7 (N-ethylcarbamic acid 9alpha-fluoro -11beta,21-dihydroxy-16betamethyl-3,20-dioxopregna

-1,4-dien-17-yl ester) is a betamethasone-derived 17alphacarbamate with selective activity on GRs. ${ }^{15}$ In A549 cells stimulated with phorbol 12-myristate 13-acetate, compound 7 showed a full transrepressor activity leading to potent reduction of matrix metalloprotease-1 levels. ${ }^{23}$ Compound 7 has never been under clinical investigation for asthma; the only in vivo results come from a study performed on rats where the administration of the molecule caused a significant anti-inflammatory effect with low impact on glucose, insulin, and triglyceride levels. ${ }^{23}$

\section{Compound A}

Compound A or CpdA (2-(4-acetoxyphenyl)-2-chloro$\mathrm{N}$-methylethylammonium chloride) is a stable analogue of the hydroxyphenylaziridine precursor present in the Namibian shrub Salsola tuberculatiformis Botschantzev. ${ }^{68}$ Although CpdA is not characterized by a classical steroidal chemical structure, it interacts with the GR and interferes with nuclear factor-kappa $B(N F-\kappa B)$-driven gene expression. $^{68}$ However, a recent study reported that CpdA modulated its anti-inflammatory action in macrophages via the autophagy receptor SQSTM1 and not by the GR, although the research was not specifically related 
to asthma. CpdA is as effective as DEX on inflammation, and it does not stimulate GRE-driven transactivation, with a potential lack of the typical AEs of ICSs. ${ }^{68,69}$

$\mathrm{CpdA}$ reduced, in a concentration-response manner, the gene and protein expression of fluticasone-resistant chemokines CCL5/RANTES and C-X3-C motif ligand-1 in airway smooth muscle (ASM) cells from healthy subjects and asthmatic patients. It also decreased the overexpression of the chemokine $\mathrm{C}-\mathrm{X}-\mathrm{C}$ motif ligand 10 (CXCL10). ${ }^{25-27} \mathrm{CpdA}$ also suppressed the production of GC-resistant chemokines via GR $\alpha$-independent mechanisms through the inhibition of interferon regulatory factor-1 (IRF-1) and the gene upregulation of DUSP1. ${ }^{25,26} \mathrm{CpdA}$ had no effect on the kinetic activation of the signal transducer and activator of transcription 5 and p38 induced by TNF- $\alpha$ /interferon-gamma (IFN- $\gamma$ ) combination in ASM cells, but it inhibited the cytokine-induced activation of IRF-1. ${ }^{24}$ In human respiratory epithelial A549 cells, combining CpdA 10 $\mu \mathrm{M}$ with DEX $10 \mathrm{nM}$ inhibited the expression of chemokine C-C motif ligand 2 (CCL2), CCL5/RANTES, TNF- $\alpha$, and intracellular adhesion molecule compared to monocomponents. ${ }^{29}$

In a predominantly Th2-driven murine model of asthma, CpdA 100-300 $\mu \mathrm{g}$ was as effective as DEX $5 \mu \mathrm{g}$ in abolishing ovalbumin-induced AHR ${ }^{37} \mathrm{CpdA}$ also counteracted mucus production, collagen deposition, goblet cell metaplasia, recruitment of eosinophils, neutrophils, dendritic cells, B cells, T cells, macrophages, and mast cells in bronchoalveolar lavage fluid (BALf). ${ }^{37}$ Furthermore, both CpdA and DEX inhibited the infiltration of Th2, Tc2, Th17, and Tc17 cells, suppressed cytokine production in BALf, and prevented the nuclear translocation of $\mathrm{NF}-\kappa \mathrm{B}$ to the I $\kappa \mathrm{B} \alpha$ promoter in the lung. ${ }^{37}$ Unlike DEX, CpdA confirmed the "dissociating" GR modulator profile as it failed to upregulate DUSP1 gene expression. ${ }^{37}$

No clinical trials on CpdA have been performed in asthmatic patients. ${ }^{9}$

Overall, CpdA differentially suppressed the expression of steroid-resistant inflammatory genes in ASM cells $^{25,26}$ and modulated the mechanisms leading to the pathogenesis of asthma. ${ }^{24-27} \mathrm{CpdA}$ is characterized by a favorable GR profile, by cooperatively suppressing inflammation while increasing the expression of specific anti-inflammatory genes. ${ }^{29}$ In a murine asthma model, CpdA inhibited airway inflammation and AHR without inducing transactivation, and the overall anti- inflammatory effect was mainly GR dependent via inhibiting NF- $\mathrm{KB}$ activity. ${ }^{37}$

\section{GSK 9027}

The SEGRA GSK9027 (N-[4-[1-(4-fluorophenyl)-1Hindazol-5-yl-3-(trifluoromethyl)phenyl]benzenesulfonamide) is a reverse sulfonamide benzyl analogue, with limited information concerning in vitro and in vivo studies. MMTV transactivation assays in human A549 epithelial cells demonstrated that GSK9027 was characterized by a high plasma protein binding compared to DEX, with a $<100$-fold reduction in potency. ${ }^{41}$

An in vitro study investigated the activity of GSK 9027 in human bronchial epithelial BEAS-2B cells transfected with 2XGRE-driven luciferase reporter plasmid either not treated or pre-treated with TNF- $\alpha{ }^{32}$ Relative to the maximally effective concentration of DEX, GSK 9027 produced lower 2XGRE reporter activation compared to fluticasone furoate (FF), FP, and BUD; therefore, it can be considered a partial agonist. ${ }^{32}$ Unlike full agonists, GSK9027 induced weak gene expression of TSC22D3 and RGS2. ${ }^{32}$

In mice challenged with IL-1, GSK9027 0.1-100 mg/ $\mathrm{kg}$ PO dose-dependently reduced circulating IL-6 levels. ${ }^{41}$ In mice and rats, GSK9027 PO produced a $t_{\max }>6.0 \mathrm{~h}$, and showed low clearance and a moderate volume of distribution, with an elimination $t_{1 / 2}$ of $3.0-4.0 \mathrm{~h}^{41}$ Evaluation of systemic exposure of GSK9027 documented a low level of unbound drug fraction in plasma, compared to DEX. ${ }^{41}$ The compound is not currently under investigation for asthma, and therefore no clinical data regarding the safety and efficacy of GSK9027 are available.

\section{GW870086X}

The SEGRA GW870086X (also called GW870086), currently under development for the treatment of asthma, is characterized by a classical steroidal structure similar to that of FF, but with a modification at position $17-\alpha{ }^{70}$ This confers enhanced affinity for the GR, leading to potent anti-inflammatory activity mediated by the ability to regulate only a subset of those genes that are normally affected by classical ICSs. ${ }^{40}$

In BEAS-2B cells transfected with 2XGRE-driven luciferase reporter plasmid, either pre-treated or not with TNF- $\alpha,{ }^{32}$ GW870086X induced lower $E_{\max }$ and 2XGRE reporter activation compared to $\mathrm{FF}, \mathrm{FP}$, and $\mathrm{BUD}$, and can thus be considered a partial agonist. ${ }^{32}$ Unlike full agonists, GW870086X only weakly induced the gene expression of TSC22D3 and regulator of RGS2. ${ }^{32}$ 
In A549 lung epithelial cells, the anti-inflammatory profile of GW870086X was similar to that of FP and antagonized the activity of DEX on MMTV-driven reporter gene transactivation. ${ }^{40}$ GW870086X induced the expression of specific genes that were also activated by other GCs, without compromising the repression of known pro-inflammatory target genes. ${ }^{40}$ In human normal 16HBE bronchial epithelial cells, GW870086X was able to strengthen tight junctions similarly to FP, although no protective effect against elastase-mediated damage was reported. ${ }^{40}$

In a Phase I RCT (NCT00483899) performed in steroid-naïve mildly asthmatic patients, GW870086X 1-3 mg via the DPI Diskhaler ${ }^{\circledR}$ produced a $C_{\max }$ of $198-207 \mathrm{pg} /$ $\mathrm{mL}$ and a $t_{\max }$ of $0.08-4.50 \mathrm{~h} .^{53,71}$ There was no reduction in total urinary free cortisol and osteocalcin after repeat dosing with GW870086X, indicating a lower potential for metabolic and bone AEs. ${ }^{53,71}$ GW870086X protected against adenosine-induced bronchoconstriction, ${ }^{53,71}$ leading to estimated mean doubling dose differences of $1.12-1.18 .^{53,71} \mathrm{GW} 870086 \mathrm{X} 1 \mathrm{mg}$, but not $3 \mathrm{mg}$, reduced $\mathrm{F}_{\mathrm{E}} \mathrm{NO}$ concentrations $2-26 \mathrm{~h}$ post-dose compared to placebo. ${ }^{53,71}$

A Phase I RCT (NCT00549497) conducted in healthy subjects assessed that inhalation of GW870086X via DPI at $15 \mathrm{mg}$, but not at 6-12 $\mathrm{mg}$, reduced the 24-hour serum cortisol level, although this reduction was not considerably different from placebo. ${ }^{59,72}$ The $t_{\max }$ of GW870086X was $0.8-3.5 \mathrm{~h}$ and $t_{1 / 2}$ was $22.7 \mathrm{~h} .^{59,72}$

According to a Phase I RCT (NCT01160003), GW870086X 5-8.75 mg was absorbed 20-25 min after administration. The $C_{\max }$ was $1212-2073 \mathrm{pg} / \mathrm{mL}, t_{\max }$ was $0.33-0.43 \mathrm{~h}$, and $t_{1 / 2}$ was $25.0-29.59 \mathrm{~h}^{58,59} \mathrm{GW} 870086 \mathrm{X}$ $5 \mathrm{mg}$ produced a partial reduction in total 24-hour serum cortisol. $^{58,59}$

In a Phase II RCT (NCT00945932), GW870086X $1 \mathrm{mg}$ administered in patients with mild to moderate asthma did not improve $\mathrm{FEV}_{1}$ or the use of rescue medication compared to placebo. ${ }^{56,57}$

A Phase II RCT (NCT01245426) investigated the treatment efficacy of GW870086X in adults with mild to moderate asthma. ${ }^{60}$ Inhaled GW870086X $2 \mathrm{mg}$ and $4 \mathrm{mg}$, but not GW870086X $3 \mathrm{mg}$, consistently improved $\mathrm{FEV}_{1}$ compared to placebo (MD 159-172 mL). ${ }^{60,63}$

In a Phase IIa RCT (NCT00857857), repeat inhalation of GW870086X 0.25-3 mg considerably improved LAR, but not allergen-induced EAR, in steroid-naïve atopic mildly asthmatic patients. GW870086X $3 \mathrm{mg}$ was as effective as FP $0.25 \mathrm{mg}$ in enhancing $\mathrm{FEV}_{1}$ (MD 229-256 mL vs placebo). ${ }^{61,64}$

In another Phase II RCT (NCT01245426), GW870086X 2-4 mg produced $C_{\max }$ values of $216-419 \mathrm{pg} / \mathrm{mL} .^{60,63}$

No safety issues were identified in patients treated with GW870086X 0.25-15 mg. ${ }^{56,59-61,63,64}$ GW870086X is not currently under investigation for asthma; the last update was posted in February 2018 and the status of the clinical trials is yet to be completed.

GW870086X is a partial agonist with weak antiinflammatory activity, ${ }^{32}$ although it protects epithelial cells from elastase-induced damage. ${ }^{40}$ In mild to moderate asthma, GW870086X reduced airway inflammation, ${ }^{53,71}$ improved lung function, ${ }^{56,57,60,63}$ and protected against allergen-induced LAR. ${ }^{61,64}$ GW870086X was rapidly absorbed, ${ }^{59,72}$ and did not produce a clinically relevant reduction in cortisol or osteocalcin. ${ }^{53,59,71,72}$ GW870086X was generally well tolerated. ${ }^{59,72}$

\section{JTPII 7968}

The SEGRA JTP117968 ((4b'S,7'R,8a'S)-4b'-benzyl-7'hydroxy-N-(2-methylpyridin-3-yl)-7'-(trifluoromethyl)-4b ',6', 7', 8',8a',10'-hexahydro-5'H-spiro[cyclopropane-1,9'phenanthrene]-2'-carboxamide) was found to have the same affinity to GRs as prednisolone, and a moderate affinity for PRs. ${ }^{21}$ JTP117968 did not bind to androgen and estrogen receptors, and, differently from prednisolone, it showed no mineralocorticoid receptor activity. ${ }^{21}$ In human A549 lung epithelial cells pre-treated with TNF$\alpha$, JTP117968 $1 \mu \mathrm{M}$ exerted a partial transrepressor activity and reduced approximately by half the production of IL-6, compared to the efficacy of prednisolone $1 \mu \mathrm{M} .{ }^{21} \mathrm{In}$ the MMTV/A549 reporter gene assay, JTP117968 demonstrated an extremely low transactivator activity compared to prednisolone. ${ }^{21}$ No further data regarding the efficacy and safety of JT117968 are currently available and the compound is not under investigation for asthma.

\section{PF802}

PF802 ((4bS,7R,8aR)-4b-benzyl-7-hydroxy-N-(2-methyl pyridin-3-yl)-7(trifluoromethyl)-4-b,5,6,7,8,8a,9,10-octa hydrophenanthrene-2-carboxamide) is an active form of the SEGRA fosdagrocorat. ${ }^{21}$ PF802 was as potent as JTP117968 in binding GRs, with a moderate binding affinity for PRs, and no binding to androgen and estrogen receptors. ${ }^{21}$ Similarly to JTP117968, PF802 had no mineralocorticoid receptor activity. ${ }^{21}$ In human A549 lung epithelial cells pre-treated with TNF- $\alpha$, PF802 demonstrated a more potent 
transrepressor activity in respect to JTP117968, leading to a greater inhibition of IL- 6 production. ${ }^{21}$ In the MMTV/A549 reporter gene assay, PF802 showed a greater transactivator activity than that elicited by JTP117968, although previous data did not confirm this finding. ${ }^{21}$ No further data regarding the efficacy and safety of PF802 in asthma are currently available and the compound is not under investigation for asthma, although there are data concerning the parent drug fosdagrocorat, which was shown to be well tolerated with an acceptable safety profile, as observed in two RCTs on rheumatoid arthritis (NCT01393639 and NCT00938587). ${ }^{73,74}$

\section{RU24858}

RU24858 (9 $\alpha$-fluoro-11 $\beta$-hydroxy-16 $\alpha$-methylpregna-21cyanide-1,4-diene-3,20-dione) is the first SEGRA to demonstrate dissociation between transactivation and transrepression. ${ }^{70}$ RU24858 elicits strong transrepressor activity by inhibiting activator protein-1, with no relevant transactivator activity compared to $\mathrm{DEX}^{70}$

RU24858 and DEX both repressed IL-1 $\beta$-induced expression of cyclooxygenase- 2 and IL-8 in A549 pulmonary cells. ${ }^{31}$ RU24858 is nearly as effective as DEX at inducing transrepression of NF- $\kappa \mathrm{B}$-dependent transcription by eliciting a lower GRE-dependent transactivator activity than DEX. ${ }^{31}$ Two further in vitro studies failed to demonstrate the full "dissociative" profile of RU24858 in human peripheral blood eosinophils and neutrophils. ${ }^{28,33}$

RU24858 $1 \mu \mathrm{M}$ induced transactivation in eosinophils by increasing the expression of chemokine receptor- 4 and annexin-I, and elicited transrepression by suppressing the production of IL-8 and monocyte chemoattractant protein1 , similarly to mometasone (MOM) $1 \mu \mathrm{M}$ and DEX $1 \mu \mathrm{M}^{28}$

RU24858 increased spontaneous eosinophil apoptosis as well as DEX and MOM, but unlike the classical GCs, RU24858 was unable to reverse IL-5- or GM-CSFinduced eosinophil survival, indicating a less effective anti-inflammatory activity. ${ }^{28}$ In human neutrophils, RU24858 increased the gene expression of growth factor receptor bound protein-2, leukotriene $\mathrm{B}_{4}$ receptor-1 and annexin-1, suppressed the production of C-X-C motif ligand 8 (CXCL8, also known as IL-8) and macrophage inflammatory protein-1 alpha, and enhanced GM-CSFinduced neutrophil survival to a similar extent to DEX. ${ }^{33}$

In human BEAS-2B cells transfected with 2XGREdriven luciferase reporter plasmid either pre-treated or not with TNF- $\alpha$, RU24858 was less effective than DEX, FF, FP, and BUD on 2XGRE reporter activation, indicating that RU24858 should be considered as a partial agonist. The effect of RU24858 on the gene expression of TSC22D3 and RGS2 was weak compared to DEX. ${ }^{32}$

In a rat cross-linked dextran gel model of lung edema, RU24858 was as effective as BUD on inflammation, although it induced systemic effects similar to classical GCs, namely a reduction in osteocalcin levels and body weight, and changes in quantitative osteopenia of the femur. $^{38}$ These data indicate that although RU24858 may have some separation between transactivation and repression in vitro, these effects are diminished in vivo and do not translate into an improved safety profile. ${ }^{38}$

No safety data are available since no clinical trials are currently ongoing.

Overall, RU24858 is effective in inducing GCinducible genes and repressing expression of inflammatory markers, ${ }^{31}$ but it did not demonstrate a fully dissociative potential between GRE-dependent transactivation and transrepression. $^{28}$ In fact, RU24858 can be considered a partial GR agonist. ${ }^{32}$ RU24858 induced the expression of specific anti-inflammatory genes and inhibited the production of pro-inflammatory mediators, ${ }^{33}$ although it was less potent than other GCs. ${ }^{28}$ The benefit/risk ratio profile of RU24858 was not superior to that of classical GCs. ${ }^{38}$

\section{TPII020}

TPI1020 (NCX 1020, NO-budesonide) is a novel antiinflammatory compound consisting of a BUD molecule linked to a nitric oxide (NO) donor. ${ }^{5}$ When topically administered in the airways, TPI1020 undergoes enzymatic cleavage to yield BUD and gaseous $\mathrm{NO}$ at the target site, thus inducing an ASM relaxant effect. ${ }^{5}$

In guinea pigs challenged with LPS, pre-treatment with inhaled TPI1020 inhibited histamine-induced AHR reduced neutrophil and myeloperoxidase levels compared to BUD. ${ }^{36}$ TPI1020 also induced concentration-dependent bronchodilation in conscious guinea pigs. ${ }^{34}$ When combined with salbutamol (SAL) $80 \mu \mathrm{M}$, TPI1020 0.11-0.33 mM elicited a greater inhibition of histamine-induced bronchoconstriction compared to either drug administered individually. ${ }^{34}$ TPI1020 $0.33 \mathrm{mM}$ plus SAL $80 \mu \mathrm{M}$ prolonged the bronchodilatory effect $(45-75 \mathrm{~min})$ compared to monocomponents. ${ }^{34}$ In another pre-clinical study on conscious guinea-pigs, only high doses of TPI1020 (0.33-0.7 $\mathrm{mM}$ ) induced short-term inhibition of histamine-induced bronchoconstriction. ${ }^{35}$ Combining TPI1020 $0.11 \mathrm{mM}$ with either formoterol $2.5 \mu \mathrm{M}$ or tiotropium $2 \mu \mathrm{M}$ increased the bronchoprotection against provocation with histamine and methacholine compared to monocomponents. ${ }^{35}$ 
According to a randomized escalated-dose study performed on smokers with mild asthma, TPI1020 had similar effects to equivalent doses of BUD on $\mathrm{FEV}_{1}$, PEF, rescuemedication use, response to methacholine, asthma control scoring system, $\mathrm{F}_{\mathrm{E}} \mathrm{NO}$, and sputum and blood eosinophils. ${ }^{62}$ TPI1020 was more effective than BUD on sputum neutrophils in patients with neutrophilia at baseline. ${ }^{62}$ Unlike BUD, TPI1020 showed no modulatory effect on $24 \mathrm{~h}$ urinary free cortisol, owing to a lower systemic corticosteroid exposure of the investigated drug. ${ }^{62}$ TPI1020 $600 \mu \mathrm{g}$ produced a $C_{\max }$ of $95.2 \mathrm{pg} / \mathrm{mL}$ and a $t_{\max }$ of $3.0 \mathrm{~h}$, whereas BUD $400 \mu \mathrm{g}$ elicited a $C_{\max }$ of $490.1 \mathrm{pg} / \mathrm{mL}$ and a $t_{\max }$ of $0.5 \mathrm{~h}^{62}$

TPI1020 was safe in mild asthmatic smoking patients, and no SAEs were recorded. ${ }^{62}$ Compared to BUD, TPI1020 caused three-fold fewer treatment-emergent AEs. ${ }^{62}$ The compound is not currently under investigation for asthma; the only study performed in asthmatic patients was a Phase II trial, with the last update posted in December 2012.

In general, TPI1020 is effective against airway inflammation and AHR, ${ }^{36}$ and when combined with a LABA, it elicited a greater bronchodilation compared to monocomponents. ${ }^{34,35}$ TPI1020 was as effective as BUD, generally safe, and characterized by a favorable PK profile with no effects on cortisol level. ${ }^{62}$

\section{Discussion}

For more than half a century, classical GCs have been considered the most important and most frequently used anti-inflammatory and immunosuppressive drugs, and they are still considered the mainstay for the treatment of asthma. Despite the efforts to improve the benefit/risk ratio of GCs, their use may be problematic owing to their safety profile, especially when administered as lifetime therapy and at high dose. Novel selective GR agonists capable of maintaining a beneficial anti-inflammatory action while reducing the risk of AEs are highly anticipated for the treatment of inflammatory disorders such as asthma.

Several compounds are currently under pre-clinical development, but only three novel experimental GR agonists (GW870086X, AZD5423, and AZD7594) seem to have some potential therapeutic relevance and have entered clinical trials for the treatment of asthma.

So far, limited success has been obtained in the development of novel GR agonists, perhaps because the hypothesis that AEs result only from transactivation and the beneficial anti-inflammatory effects from transrepression seems to be oversimplified. Indeed, identifying a fully dissociated GR agonist showing exclusively transrepressive activity without transactivation turned out to be challenging. It was documented that transactivation activities rely strongly on GR levels, co-repressors, and co-activators, and are cell type and tissue dependent. ${ }^{75}$ For example, the activity of RU24858 was found to be highly dependent on the level of GR expression and any increase in the coactivators could stabilize the RU24858/GR complex, changing RU24858 into a potent agonist. ${ }^{75}$ Conversely, the overexpression of the co-repressor silencing mediator of retinoid and thyroid receptor led to a reduction in the transcriptional potency of RU24858. ${ }^{75}$

Another critical point regards the real binding selectivity of SEGRAs for the GR, which has a common protein structure with other steroidal receptors such as mineralocorticoid, estrogen, and androgen receptors. Although A276575 had high repressive and very low transactivator activities, it failed to show an effective anti-inflammatory action owing to the high affinity for PRs, which translated into undesirable AEs. ${ }^{22}$ This evidence highlights the relevance of thorough drug design in order to minimize offtarget AEs due to scarce selectivity, while increasing GR therapeutic effects. $^{76}$

On the other hand, GW870086X, RU24858, and GSK9027 exhibited a partial agonistic activity on GRE reporters and a weak gene expression of TSC22D3 and RGS2, which is required for the beneficial therapeutic activities of GCs. ${ }^{32}$ Therefore, it has been hypothesized that, on the one hand, partial agonists may exert suboptimal biological effects compared to those exerted by classical GCs owing to their reduced gene expression profile, while on the other hand, this feature may have conferred SEGRAs with the ability to induce fewer AEs, given that genes responsible for AEs are induced in a ligand efficacy-dependent manner. ${ }^{32}$ This could translate into an optimized strategy of drug delivery, with full agonists such as classical GCs being used as topical drugs via inhalation, and partial agonists administered systemically, where AEs represent a major concern. ${ }^{40,56}$

In any case, it seems very difficult to really uncouple the therapeutic and harmful effects mediated by GRs. AZD7594 is the most recent non-steroidal GR agonist known to have progressed to Phase II studies for the treatment of asthma. Considering that the last Phase II trial on GW870086X terminated in 2011, AZD7594 and AZD5423 could be considered the only two experimental selective GR agonists in active clinical development for asthmatic patients. Among the newly discovered 
compounds, JTP117968 exhibited improved transrepressor versus transactivator activity with no mineralocorticoid effects. Thus, it is expected that JTP117968 could be a suitable compound for developing ideal SEGRAs in the future. Also, GSK9027 demonstrated a potent transrepressor action, with a pharmacological profile similar to DEX, despite a low potency due to the high protein binding. Further larger clinical trials are needed to confirm the dissociated nature of currently developed SEGRAs and their potential superiority over traditional GCs.

\section{Conclusion}

SEGRAs capable of optimizing genomic GC effects by preferentially inducing transrepression over transactivation remain a challenging matter to be further investigated for the treatment of asthma. Since data from pre-clinical studies have been not always been confirmed in clinical investigations, well-designed RCTs are needed in asthmatic patients to confirm the potentially positive benefit/risk ratio of each specific SEGRA and to optimize the development strategy of these agents in respiratory medicine.

\section{Abbreviations}

AE, adverse event; AHR, airway hyperresponsiveness; ASM, airway smooth muscle; BALf, bronchoalveolar lavage fluid; BUD, budesonide; CCL2, C-C motif ligand 2; CCL5, $\mathrm{C}-\mathrm{C}$ motif ligand 5 (also known as RANTES); $C_{\max }$, maximum drug concentration; CXCL8, C-X-C motif ligand 8 (also known as IL-8); CXCL10, C-X-C motif ligand 10; DEX, dexamethasone; DPI, dry powder inhaler; DUSP1, dual-specificity phosphatase-1 (also known as mitogenactivated protein kinase phosphatase-1 [MKP1]); EAR, early asthmatic response; $E_{\max }$, maximal response of efficacy; $\mathrm{F}_{\mathrm{E}} \mathrm{NO}$, fraction exhaled of nitric oxide; $\mathrm{FEV}_{1}$, forced expiratory volume in 1 second; FF, fluticasone furoate; FP, fluticasone propionate; GC, glucocorticoid; GM-CSF, granulocyte-macrophage colony-stimulating factor; GR, glucocorticoid receptor; GRE, glucocorticoid response element; ICS, inhaled corticosteroid; IFN- $\gamma$, interferon gamma; IL, interleukin; IRF-1, interferon regulatory factor-1; IV, intravenous administration; LABA, long-acting $\beta_{2}$-adrenoceptor agonist; LAR, late asthmatic response; LPS, lipopolysaccharide; MD, mean difference; MMTV, mouse mammary tumor virus; MOM, mometasone; NF- $\kappa \mathrm{B}$, nuclear factorkappa B; NO, nitric oxide; PD, pharmacodynamics; PEF, peak expiratory flow; PICO, patient problem, intervention, comparison, and outcome; PK, pharmacokinetics; pMDI, pressurized metered-dose inhaler; PO, per os; PR, progesterone receptor; PRISMA-P, preferred reporting items for systematic reviews and meta-analyses protocols; RANTES, regulated on T-cell activation, normal T-cell expressed and secreted; RCT, randomized controlled trial; RGS2, regulator of G protein signaling 2; RoB2, Risk of Bias 2; SAE, serious adverse event; SAL, salbutamol; SEGRA, selective glucocorticoid receptor agonist; SEGRM, selective glucocorticoid receptor modulator; $t_{1 / 2}$, half-life; $t_{\max }$, time to reach $C_{\text {max }}$; TNF- $\alpha$, tumor necrosis factor-alpha; TSC22D3, TSC22 domain family member 3 (also known as glucocorticoid-induced leucine zipper).

\section{Disclosure}

PR reports grants and personal fees from Boehringer Ingelheim, grants and personal fees from Novartis, personal fees from AstraZeneca, grants and personal fees from Chiesi Farmaceutici, grants and personal fees from Almirall, grants from Zambon, personal fees from Biofutura, personal fees from GlaxoSmithKline, personal fees from Menarini, and personal fees from Mundipharma. $\mathrm{MC}$ has participated as a faculty member and advisor in scientific meetings and courses under the sponsorship of Almirall, AstraZeneca, Biofutura, Boehringer Ingelheim, Chiesi Farmaceutici, GlaxoSmithKline, Menarini Group, Lallemand, Mundipharma, Novartis, Pfizer, Verona Pharma, and Zambon; is or has been a consultant to ABC Farmaceutici, AstraZeneca, Chiesi Farmaceutici, Edmond Pharma, Lallemand, Novartis, Ockham Biotech, Verona Pharma, and Zambon, and his department was funded by Almirall.

LC reports grants and personal fees from Boehringer Ingelheim, grants and personal fees from Novartis, nonfinancial support from AstraZeneca, grants from Chiesi Farmaceutici, grants from Almirall, personal fees from ABC Farmaceutici, personal fees from Edmond Pharma, grants and personal fees from Zambon, personal fees from Verona Pharma, and personal fees from Ockham Biotech. The authors report no other conflicts of interest in this work.

\section{References}

1. Quirce S, Bobolea I, Barranco P. Emerging drugs for asthma. Expert Opin Emerg Drugs. 2012;17(2):219-237. doi:10.1517/14728214. 2012.683410

2. Olin JT, Wechsler ME. Asthma: pathogenesis and novel drugs for treatment. BMJ. 2014;349:g5517. doi:10.1136/bmj.g5517

3. Stoloff SW, Kelly HW. Updates on the use of inhaled corticosteroids in asthma. Curr Opin Allergy Clin Immunol. 2011;11(4):337-344. doi:10.1097/ACI.0b013e328348a813 
4. Hoes JN, Jacobs JW, Buttgereit F, Bijlsma JW. Current view of glucocorticoid co-therapy with DMARDs in rheumatoid arthritis. Nat Rev Rheumatol. 2010;6(12):693. doi:10.1038/nrrheum.20 10.179

5. Westergaard CG, Porsbjerg C, Backer V. Emerging corticosteroid agonists for the treatment of asthma. Expert Opin Emerg Drugs. 2015;20(4):653-662. doi:10.1517/14728214.2015.1061503

6. Strehl C, Buttgereit F. Optimized glucocorticoid therapy: teaching old drugs new tricks. Mol Cell Endocrinol. 2013;380(1-2):32-40. doi:10.1016/j.mce.2013.01.026

7. Gerber AN. Measuring safety of inhaled corticosteroids in asthma. Ann Allergy Asthma Immunol. 2016;117(6):577-581. doi:10.1016/j.anai.2016.05.028

8. Schacke H, Schottelius A, Docke WD, et al. Dissociation of transactivation from transrepression by a selective glucocorticoid receptor agonist leads to separation of therapeutic effects from side effects. Proc Natl Acad Sci U S A. 2004;101(1):227-232. doi:10.1073/pnas.0300372101

9. Sundahl N, Bridelance J, Libert C, De Bosscher K, Beck IM. Selective glucocorticoid receptor modulation: new directions with non-steroidal scaffolds. Pharmacol Ther. 2015;152:28-41. doi:10.1016/j.pharmthera.2015.05.001

10. De Bosscher K, Beck IM, Ratman D, Berghe WV, Libert C. Activation of the glucocorticoid receptor in acute inflammation: the SEDIGRAM concept. Trends Pharmacol Sci. 2016;37(1):4-16. doi:10.1016/j.tips.2015.09.002

11. Moher D, Shamseer L, Clarke M, et al. Preferred reporting items for systematic review and meta-analysis protocols (PRISMA-P) 2015 statement. Syst Rev. 2015;4:1. doi:10.1186/2046-4053-4-1

12. Schardt C, Adams MB, Owens T, Keitz S, Fontelo P. Utilization of the PICO framework to improve searching PubMed for clinical questions. BMC Med Inform Decis Mak. 2007;7:16. doi:10.1186/ 1472-6947-7-16

13. Van Norman GA. Drugs and devices: comparison of European and U.S. approval processes. JACC Basic Transl Sci. 2016;1(5):399-412. doi:10.1016/j.jacbts.2016.06.003

14. European Medicines Agency. Guideline for good clinical practice E6 (R2); 2016. Available from: https://www.ema.europa.eu/en/docu ments/scientific-guideline/ich-e-6-r2-guideline-good-clinical-practice -step-5_en.pdf. Accessed April 24, 2020.

15. Gessi S, Merighi S, Andrea Borea P. Glucocorticoids' pharmacology: past, present and future. Curr Pharm Des. 2010;16(32):3540-3553. doi: $10.2174 / 138161210793797915$

16. Lesovaya E, Yemelyanov A, Swart AC, Swart P, Haegeman G, Budunova I. Discovery of Compound A-a selective activator of the glucocorticoid receptor with anti-inflammatory and anti-cancer activity. Oncotarget. 2015;6(31):30730-30744. doi:10.18632/oncotarget.5 078

17. Luypaert A, Berghe WV, Tavernier J, Libert C, De Bosscher K. Strategies and compounds to circumvent glucocorticoid-induced side effects. In: Riccardi C, Levi-Schaffer F, Tiligada E, editors. Immunopharmacology and Inflammation. Springer, Cham; 2018:283-305.

18. Meijer OC, Koorneef LL, Kroon J. Glucocorticoid receptor modulators. Annales d'endocrinologie. 2018;79(3). doi:10.1016/j. ando.2018.03.004

19. Jadad AR, Moore RA, Carroll D, et al. Assessing the quality of reports of randomized clinical trials: is blinding necessary? Control Clin Trials. 1996;17(1):1-12. doi:10.1016/0197-2456(95) 00134-4

20. Higgins JPT, Savović J, Page MJ, Elbers RG, Sterne JAC Chapter 8: assessing risk of bias in a randomized trial. Cochrane handbook for systematic reviews of interventions version 6.0 (updated July 2019). Cochrane; 2019. Available from: http://www.training.cochrane.org/hand book. Accessed July 3, 2020.

21. Kurimoto T, Tamai I, Miyai A, et al. JTP-117968, a novel selective glucocorticoid receptor modulator, exhibits improved transrepression/ transactivation dissociation. Eur J Pharmacol. 2017;803:179-186. doi:10.1016/j.ejphar.2017.03.057
22. Lin CW, Nakane M, Stashko M, et al. Trans-Activation and repression properties of the novel nonsteroid glucocorticoid receptor ligand 2, 5-dihydro-9-hydroxy-10-methoxy-2, 2, 4-trimethyl-5-(1-methylcyclohexen-3-y1)-1H-[1] benzopyrano [3, 4-f] quinoline (A276575) and its four stereoisomers. Mol Pharmacol. 2002;62(2):297-303. doi:10.1124/mol.62.2.297

23. Ali A, Balkovec JM, Greenlee M, et al. Discovery of betamethasone $17 \alpha-$ carbamates as dissociated glucocorticoid receptor modulators in the rat. Bioorg Med Chem. 2008;16(16):7535-7542. doi:10.1016/j.bmc.2008.07.037

24. Gavrila A, Chachi L, Tliba O, Brightling CE, Amrani Y. Compound A (CpdA) supressed production of corticosteroid-resistant chemokines via GR-independent pathways including the inhibition of IRF-1 in airway smooth muscle (ASM) cells. In: D29. molecular signals and cellular mechanics: focus on asthma. American Thoracic Society; 2014:A5605A5605.

25. Gavrila A, Chachi L, Tliba O, Brightling C, Amrani Y. Effect of the plant derivative Compound A on the production of corticosteroid-resistant chemokines in airway smooth muscle cells. Am J Respir Cell Mol Biol. 2015;53(5):728-737. doi:10.1165/rcmb.2014-0477OC

26. Gavrila A. Modulating Steroid Insensitive Pathways in Airway Smooth Muscle Cells; Thesis Submitted for the Degree of Doctor of Philosophy at the University of Leicester: Department of Infection, Immunity and Inflammation. University of Leicester; 2016.

27. Chachi L, Gavrila A, Tliba O, Brightling C, Amrani Y. The Dissociated Steroid Receptor Ligand from Plant Origin Called Compound A (CpdA) Inhibits the Production of Steroid-Resistant Chemokines Induced by TNFa IFNy in Airway Smooth Muscle (ASM) Cells in Both Asthma and Healthy Subjects. European Respiratory Society; 2012.

28. Janka-Junttila M, Moilanen E, Hasala H, Zhang X, Adcock I, Kankaanranta H. The glucocorticoid RU24858 does not distinguish between transrepression and transactivation in primary human eosinophils. J Inflam (Lond). 2006;3:10. doi:10.1186/1476-92553-10

29. Desmet SJ, Bougarne N, Van Moortel L, et al. Compound A influences gene regulation of the Dexamethasone-activated glucocorticoid receptor by alternative cofactor recruitment. Sci Rep. 2017;7 (1):8063. doi:10.1038/s41598-017-07941-y

30. McWhae A, Shah S, Karrman-Mardh C, Miller-Larsson A, Giembycz M, Newton R. The novel glucocorticoid receptor agonist, AZD5423, shows super-agonism relative to the conventional agonists, dexamethasone and budesonide. In: A31. the epithelium in lung inflammation. American Thoracic Society; 2015:A1293A1293.

31. Chivers JE, Gong W, King EM, et al. Analysis of the dissociated steroid RU24858 does not exclude a role for inducible genes in the anti-inflammatory actions of glucocorticoids. Mol Pharmacol. 2006;70(6):2084-2095. doi:10.1124/mol.106.025841

32. Rider CF, Shah S, Miller-Larsson A, Giembycz MA, Newton R, Wang Z. Cytokine-induced loss of glucocorticoid function: effect of kinase inhibitors, long-acting $\beta$ (2)-adrenoceptor [corrected] agonist and glucocorticoid receptor ligands. PLoS One. 2015;10(1): e0116773-e0116773. doi:10.1371/journal.pone.0116773

33. Janka-Junttila M, Hasala H, Adcock I, Moilanen E, Kankaanranta H. Dexamethasone and RU24858 induce survival and growth factor receptor bound protein 2, leukotriene B4 receptor 1 and annexin-1 expression in primary human neutrophils. $J$ Cell Death. 2012;5:21-29. doi:10.4137/JCD.S9097

34. Turner DL, Ferrari N, Ford WR, et al. TPI 1020, a novel anti-inflammatory, nitric oxide donating compound, potentiates the bronchodilator effects of salbutamol in conscious guinea-pigs. Eur J Pharmacol. 2010;641(23):213-219. doi:10.1016/j.ejphar.2010.05.025

35. Turner D, Ferrari N, Ford WR, et al. Bronchoprotection in conscious guinea pigs by budesonide and the NO-donating analogue, TPI 1020, alone and combined with tiotropium or formoterol. Br J Pharmacol. 2012;167(3):515-526. doi:10.1111/j.1476-5381.2012.02016.x 
36. Nevin BJ, Broadley KJ. Comparative effects of inhaled budesonide and the NO-donating budesonide derivative, NCX 1020, against leukocyte influx and airway hyperreactivity following lipopolysaccharide challenge. Pulm Pharmacol Ther. 2004;17(4):219-232. doi:10.1016/j.pupt.2004.04.002

37. Reber LL, Daubeuf F, Plantinga M, et al. A dissociated glucocorticoid receptor modulator reduces airway hyperresponsiveness and inflammation in a mouse model of asthma. J Immunol. 2012;188 (7):3478-3487. doi:10.4049/jimmunol.1004227

38. Belvisi MG, Wicks SL, Battram CH, et al. Therapeutic benefit of a dissociated glucocorticoid and the relevance of in vitro separation of transrepression from transactivation activity. J Immunol. 2001;166 (3):1975-1982. doi:10.4049/jimmunol.166.3.1975

39. Mardh CK, Russell W, Gustavsson M, et al. A novel inhaled nonsteroidal modulator of inflammation for the control of asthma; AZD7594. In: A31. Asthma Therapy: Glucocorticoids and Beyond. American Thoracic Society; 2016:A1329-A1329.

40. Uings IJ, Needham D, Matthews J, et al. Discovery of GW870086: a potent anti-inflammatory steroid with a unique pharmacological profile. Br J Pharmacol. 2013;169(6):1389-1403. doi:10.1111/bph. 12232

41. Yates CM, Brown PJ, Stewart EL, et al. Structure guided design of 5-arylindazole glucocorticoid receptor agonists and antagonists. J Med Chem. 2010;53(11):4531-4544. doi:10.1021/ jm100447c

42. Coghlan MJ, Kym PR, Elmore SW, et al. Synthesis and characterization of non-steroidal ligands for the glucocorticoid receptor: selective quinoline derivatives with prednisolone-equivalent functional activity. J Med Chem. 2001;44(18):2879-2885. doi:10.1021/jm01 $0228 \mathrm{c}$

43. NCT02479412. A multiple dosing (14 days) study to assess efficacy and safety of three dose levels of AZD7594, given once daily by inhalation, in patients with mild to moderate asthma; 2017. Available from: https:// www.clinicaltrials.gov/ct2/show/NCT02479412. Accessed December 11,2019

44. Brown MN, Fuhr R, Beier J, et al. Efficacy and safety of AZD7594, an inhaled non-steroidal selective glucocorticoid receptor modulator, in patients with asthma: a phase 2 a randomized, double blind, placebo-controlled crossover trial. Respir Res. 2019;20(1):37. doi:10.1186/s12931-019-1000-7

45. NCT02645253. A study to assess the safety, tolerability and pharmacokinetics of AZD7594 inhaled formulation in healthy Japanese men; 2016. Available from: https://clinicaltrials.gov/ct2/show/ NCT02645253. Accessed December 11, 2019.

46. Prothon S, Wahlby Hamren U, Tehler U, et al. Safety, pharmacokinetics and pharmacodynamics of the selective glucocorticoid receptor modulator AZD7594, following inhalation in healthy Japanese volunteers. Drug Des Devel Ther. 2019;13:3845-3853. doi:10.2147/ DDDT.S215170

47. NCT01310322. A study in healthy subjects and mild asthmatics to investigate pharmacokinetics of AZD5423 when administered in different ways; 2011. Available from: https://clinicaltrials.gov/ct2/ show/NCT01310322. Accessed July 3, 2020.

48. Melin J, Prothon S, Kloft C, et al. Pharmacokinetics of the inhaled selective glucocorticoid receptor modulator AZD5423 following inhalation using different devices. AAPS J. 2017;19(3):865-874. doi:10.1208/s12248-016-0042-8

49. NCT01635985. A study in healthy subjects to investigate pharmacokinetics of AZD5423 when administered in different ways; 2012. Available from: https://clinicaltrials.gov/ct2/show/NCT01635985. Accessed December 16, 2019.

50. Werkstrom V, Prothon S, Ekholm E, Jorup C, Edsbacker S. Safety, pharmacokinetics and pharmacodynamics of the selective glucocorticoid receptor modulator AZD5423 after inhalation in healthy volunteers. Basic Clin Pharmacol Toxicol. 2016;119(6):574-581. doi:10.1111/bcpt.12621
51. NCT02648438. A study to assess the bioavailability and to compare the pharmacokinetics of AZD7594 inhaled via monodose inhaler and multiple-dose dry powder inhalers (DPI) or pressurized metered-dose inhaler (pMDI) in healthy male subjects; 2016. Available from: https://clinicaltrials.gov/ct2/show/NCT02648438. Accessed December 11, 2019.

52. Chen Y, Prothon S, Eriksson U, et al. Pharmacokinetics (PK) of a Single Dose AZD7594 Administered Intravenously (IV), Orally, and Inhaled via Two Dry Powder Inhalers (DPI) and a Pressurized Metered-Dose Inhaler (pMDI). European Respiratory Society; 2017.

53. Leaker BR, O'Connor B, Singh D, Barnes PJ. The novel inhaled glucocorticoid receptor agonist GW870086X protects against adenosine-induced bronchoconstriction in asthma. J Allergy Clin Immunol. 2015;136(2):501-502.e506. doi:10.1016/j.jaci.2015.01.034

54. Gauvreau GM, Boulet LP, Leigh R, et al. A nonsteroidal glucocorticoid receptor agonist inhibits allergen-induced late asthmatic responses. Am J Respir Crit Care Med. 2015;191(2):161-167. doi:10.1164/rccm.201404-0623OC

55. NCT01225549. The study will evaluate the efficacy of AZD5423 in patients with mild asthma challenged with an inhaled allergen (allergen); 2010. Available from: https://clinicaltrials.gov/ct2/show/ NCT01225549. Accessed December 11, 2019.

56. Bareille P, Hardes K, Donald AC. Efficacy and safety of once-daily GW870086 a novel selective glucocorticoid in mild-moderate asthmatics: a randomised, two-way crossover, controlled clinical trial. $J$ Asthma. 2013;50(10):1077-1082. doi:10.3109/02770903.2013.837480

57. NCT00945932. A study to evaluate the effect of repeat doses of GW870086X in mild to moderate asthmatics; 2009. Available from: https://clinicaltrials.gov/ ct2/show/NCT00945932. Accessed December 16, 2019.

58. NCT01160003. A randomised, double-blind, placebo-controlled, dose ascending, 3-way crossover study to assess the pharmacokinetics, safety and tolerability of repeat inhaled doses of nebulised GW870086X in healthy adult male volunteers; 2010. Available from: https://clinical trials.gov/ct2/show/NCT01160003. Accessed December 11, 2019.

59. Allen A, Bareille P, Hardes K, Robertson J. Safety, tolerability, pharmacokinetics and pharmacodynamics of single and repeat doses of GW870086: two randomised studies. Curr Drug Ther. 2013;8 (2):76-85. doi:10.2174/15748855113089990004

60. NCT01245426. A phase II randomized, double-blind, placebo-controlled, parallel group, multicentre study to determine the efficacy and dose response of repeat inhaled doses of GW870086X on FEV1 in adults with persistent asthma; 2010. Available from: https://clinicaltrials.gov/ct2/show/NCT01245426. Accessed December 11, 2019.

61. NCT00857857. A randomised, placebo-controlled, incomplete block, three-way crossover study to evaluate the effect of treatment with repeat inhaled doses of GW870086 on the allergen-induced early and late asthmatic response in subjects with mild asthma; 2009. Available from: https://clinicaltrials.gov/ct2/show/NCT00857857. Accessed December 11, 2019.

62. Boulet LP, Lemiere C, Gauvreau G, et al. Safety, pharmacodynamics and pharmacokinetics of TPI 1020 in smokers with asthma. Respir Med. 2009;103(8):1159-1166. doi:10.1016/j.rmed.2009.02.011

63. Bareille P, Hardes K, Robertson J, Davis A, Allen A. Efficacy of a new selective steroid (GW870086) in asthma: an adaptive, randomised, controlled trial. Curr Drug Ther. 2013;8(2):69-75. doi:10.2174/1574885511308020001

64. Bareille P, Allen A, Hardes K, Donald A. Effect of a novel selective inhaled steroid on the allergen-induced early and late asthmatic response in adults with mild asthma: a randomised study. Curr Drug Ther. 2013;8(3):155-163. doi:10.2174/15748855113089990005

65. Elmore SW, Coghlan MJ, Anderson DD, et al. Nonsteroidal selective glucocorticoid modulators: the effect of C-5 alkyl substitution on the transcriptional activation/repression profile of 2, 5-dihydro-10methoxy-2, 2, 4-trimethyl-1H-[1] benzopyrano [3, 4-f] quinolines. J Med Chem. 2001;44(25):4481-4491. doi:10.1021/jm010367u 
66. Edman K, Ahlgren R, Bengtsson M, et al. The discovery of potent and selective non-steroidal glucocorticoid receptor modulators, suitable for inhalation. Bioorg Med Chem Lett. 2014;24(11):2571-2577. doi:10.1016/j.bmcl.2014.03.070

67. NCT03976869. A study to assess the pharmacokinetics (PK), pharmacodynamics (PD) and safety of 2-week treatment with inhaled AZD7594 in adolescents (12 to 17 years) with asthma; 2020. Available from: https://clinicaltrials.gov/ct2/show/NCT03976869? cond=AZD7594\&draw=2\&rank=5. Accessed June 23, 2020.

68. De Bosscher K, Vanden Berghe W, Beck IM, et al. A fully dissociated compound of plant origin for inflammatory gene repression. Proc Natl Acad Sci U S A. 2005;102(44):15827-15832. doi:10.1073/ pnas.0505554102

69. Dewint P, Gossye V, De Bosscher K, et al. A plant-derived ligand favoring monomeric glucocorticoid receptor conformation with impaired transactivation potential attenuates collagen-induced arthritis. J Immunol. 2008;180(4):2608-2615. doi:10.4049/jimmunol.180.4.2608

70. Cazzola M, Coppola A, Rogliani P, Matera MG. Novel glucocorticoid receptor agonists in the treatment of asthma. Expert Opin Investig Drugs. 2015;24(11):1473-1482. doi:10.1517/13543784.2015.1078310

71. NCT00483899. Examine the effect of repeat inhaled doses of GW870086X on lung function in mild asthmatic male subjects; 2007. Available from: https://clinicaltrials.gov/ct $2 /$ show/ NCT00483899. Last accessed December 16, 2019.
72. NCT00549497. A randomized study evaluating steroid hormone levels, safety and tolerability of GW870086X in healthy volunteers; 2007. Available from: https://clinicaltrials.gov/ct2/show/ NCT00549497. Last accessed December 11, 2019.

73. Buttgereit F, Strand V, Lee EB, et al. Fosdagrocorat (PF-04171327) versus prednisone or placebo in rheumatoid arthritis: a randomised, double-blind, multicentre, phase IIb study. RMD Open. 2019;5(1): e000889. doi:10.1136/rmdopen-2018-000889

74. Stock T, Fleishaker D, Wang X, Mukherjee A, Mebus C. Improved disease activity with fosdagrocorat (PF-04171327), a partial agonist of the glucocorticoid receptor, in patients with rheumatoid arthritis: a phase 2 randomized study. Int J Rheum Dis. 2017;20(8):960-970. doi:10.1111/1756-185X.13053

75. Dezitter X, Fagart J, Taront S, et al. A structural explanation of the effects of dissociated glucocorticoids on glucocorticoid receptor transactivation. Mol Pharmacol. 2014;85(2):226-236. doi:10.1124/mol.113.085860

76. Kohn JA, Deshpande K, Ortlund EA. Deciphering modern glucocorticoid cross-pharmacology using ancestral corticosteroid receptors. J Biol Chem. 2012;287(20):16267-16275. doi:10.1074/jbc.M112.346411

\section{Publish your work in this journal}

The Journal of Experimental Pharmacology is an international, peerreviewed, open access journal publishing original research, reports, reviews and commentaries on all areas of laboratory and experimental pharmacology. The manuscript management system is completely online and includes a very quick and fair peer-review system. Visit http://www.dovepress.com/testimonials.php to read real quotes from published authors. 